\title{
Rapid single-molecule detection of COVID-19 and MERS antigens via nanobody-functionalized organic electrochemical transistors
}

\author{
Keying Guo ${ }^{1,8}$, Shofarul Wustoni ${ }^{1,8}$, Anil Koklu1,8, Escarlet Díaz-Galicia ${ }^{1}{ }^{1,2}$, Maximilian Moser ${ }^{3}{ }^{3}$, \\ Adel Hama', Ahmed A. Alqahtani ${ }^{4}$, Adeel Nazir Ahmad ${ }^{5}{ }^{5}$, Fatimah Saeed Alhamlan ${ }^{4}$,

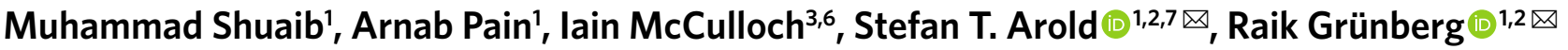 \\ and Sahika Inal ${ }^{1 凶}$
}

The coronavirus disease 2019 (COVID-19) pandemic has highlighted the need for rapid and sensitive protein detection and quantification in simple and robust formats for widespread point-of-care applications. Here, we report on nanobody-functionalized organic electrochemical transistors with a modular architecture for the rapid quantification of single-molecule-to-nanomolar levels of specific antigens in complex bodily fluids. The sensors combine a solution-processable conjugated polymer in the transistor channel and high-density and orientation-controlled bioconjugation of nanobody-SpyCatcher fusion proteins on disposable gate electrodes. The devices provide results after $10 \mathrm{~min}$ of exposure to $5 \mu \mathrm{l}$ of unprocessed samples, maintain high specificity and single-molecule sensitivity in human saliva and serum, and can be reprogrammed to detect any protein antigen if a corresponding specific nanobody is available. We used the sensors to detect green fluorescent protein, and severe acute respiratory syndrome coronavirus-2 (SARS-CoV-2) and Middle East respiratory syndrome coronavirus (MERS-CoV) spike proteins, and for the COVID-19 screening of unprocessed clinical nasopharyngeal swab and saliva samples with a wide range of viral loads.

S evere acute respiratory syndrome coronavirus 2 (SARS-CoV-2) had caused 2.5 million fatalities as of February 2021 (https:// covid19.who.int) and may remain a worldwide health and economic burden for several years ${ }^{1}$. Owing to airborne transmission ${ }^{2}$ and a large share of symptom-free but infective carriers ${ }^{3,4}$, this virus defies traditional suppression measures that are based on the isolation of symptomatic individuals. Molecular testing has, therefore, become an essential tool in global attempts to curb the coronavirus disease 2019 (COVID-19) pandemic ${ }^{5}$. Testing currently relies on PCR with reverse transcription (RT-PCR) - a well-established diagnostic method with close to single-molecule sensitivity ${ }^{6}$. However, RT-PCR requires complex sample processing in specialized, well-instrumented laboratories. Owing to the limited capacity of these laboratories and the required logistics, results are typically communicated one to several days after sample collection. This delay in isolating infected carriers is severely hampering the suppression of infection chains ${ }^{7}$.

In a point-of-care (POC) setting, lateral flow immunoassays could offer an alternative as they detect viral protein (rather than RNA) directly in unprocessed patient samples and give qualitative results within 15-30 min. Yet, fast immunoassays detect viral loads above 50 million copies per $\mathrm{ml}$, which is useful for the isolation of highly infectious individuals but not sensitive enough for diagnostic purposes $^{8}$. In contrast, enzymatic nucleic acid detection-based POC assays generally have to trade off sensitivity and robustness against sample processing and reaction times ${ }^{9}$. Thus, the COVID-19 crisis highlights a critical limitation of our current POC diagnostics toolbox. What has remained elusive so far is rapid protein detection at the single-molecule level in a format that is simple and robust enough for widespread POC or bedside application. Here, we present a bioelectronic sensor technology that fulfils these requirements.

Recent years have seen major progress in the development of transistors that transduce and, importantly, amplify biological reactions or binding events into an electrical readout ${ }^{10-14}$. Organic electrochemical transistors (OECTs) have emerged as alternative bioelectronic transducers that outperform all other electrolyte-gated transistors and perform favorably compared with most solid-state technologies, which themselves have record high transconductance values ${ }^{15}$. OECTs operate in aqueous media and integrate ion-permeable conjugated polymers in their channel, which are volumetrically (de)doped by electrolyte ions injected by a gate electrode. The volumetric coupling between ionic and electronic charges in the channel makes OECTs powerful voltage amplifiers. As such, even a few binding events at the gate electrode can cause large modulations in the channel current ${ }^{16-18}$. This efficient on-site amplification of input signal allows for miniaturization and low-noise recordings, which should make OECTs particularly suitable for POC applications ${ }^{17}$. Despite these advantages, there are only

'Biological and Environmental Science and Engineering Division, King Abdullah University of Science and Technology (KAUST), Thuwal, Saudi Arabia. ${ }^{2}$ Computational Bioscience Research Center (CBRC), Biological and Environmental Science and Engineering Division, KAUST, Thuwal, Saudi Arabia. ${ }^{3}$ Department of Chemistry, University of Oxford, Oxford, UK. ${ }^{4}$ Department of Infection and Immunity, King Faisal Specialist Hospital and Research Center, Riyadh, Saudi Arabia. ${ }^{5}$ KAUST Health, Thuwal, Saudi Arabia. ${ }^{6} \mathrm{KAUST}$ Solar Center (KSC), Physical Science and Engineering Division, KAUST, Thuwal, Saudi Arabia. ${ }^{7}$ Centre de Biochimie Structurale, CNRS, INSERM, Université de Montpellier, Montpellier, France. ${ }^{8}$ These authors contributed equally: Keying Guo,

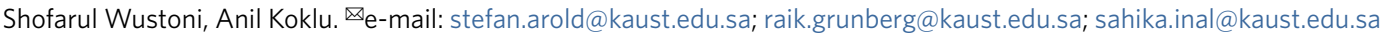


a
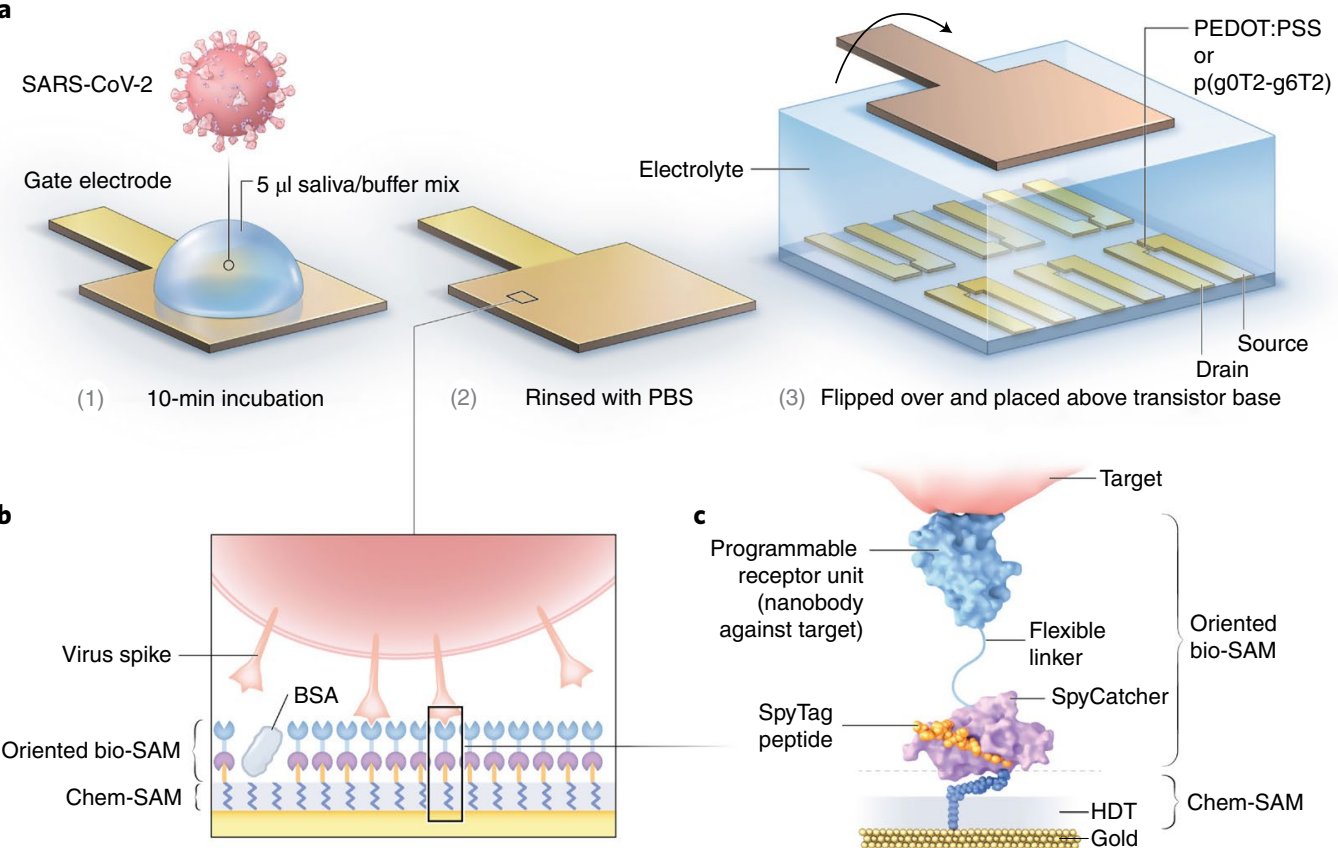

Fig. 1 Schematic of the nanobody-functionalized OECT sensor. a, Operation. The gate electrode is exposed to a mix of sample (such as saliva) and binding buffer (1), rinsed with PBS (2) and mounted on top of the OECT channel for signal acquisition (3). b, Gate functionalization layers. Chem-SAMs and bio-SAMs are self-assembled on the gate electrode surface. c, Molecular architecture. A synthetic SpyTag peptide is chemically coupled to the HDT monolayer to form a chem-SAM. The nanobody-SpyCatcher fusion protein then attaches itself to this chemical layer through the autocatalytic formation of a covalent SpyCatcher-SpyTag bond, forming the bio-SAM. The nanobody domain defines sensor specificity and is interchangeable.

a few reports of label-free OECT immunosensors ${ }^{19,20}$. More generally, current electronic protein sensors appear to translate poorly into real-world applications. Common drawbacks include complex sensor designs, the need for tightly controlled environments, reliance on regular off-the-shelf antibodies and their chemical immobilization in random orientations ${ }^{21}$.

We overcame these limitations in protein detection with a bioelectronic approach that advances OECT technology on several levels, ranging from chemical materials to biological recognition (Fig. 1). Key features in this process were: (1) the use of a novel organic semiconductor that allowed for a practical implementation of an accumulation-mode OECT; and (2) the controlled biofunctionalization of the sensor surface with (3) custom-engineered nanobodies $^{22}$. The accumulation-mode OECT combined high output signal strength and stability with very low input power requirement. The bioassembly strategy coupled recombinant proteins in a precisely defined orientation at very high physical density.

We demonstrate that the resulting sensor is fast $(<15 \mathrm{~min}$ from sample to result) and works with unprocessed saliva or serum samples. The sensor is largely reusable, easy to manufacture and highly modular. It reliably and specifically detects single protein molecules on millimeter-sized disposable electrodes in uncontrolled, ambient environments. The sensor is also quantitative and distinguishes concentrations over eight to ten orders of magnitude (attomolar to nanomolar). Experience with three different target proteins shows that our sensor technology is broadly applicable and only limited by the availability of antigen-specific nanobodies. We validated the performance with clinical unprocessed nasopharyngeal swab and saliva samples from patients with COVID-19 and demonstrate a sensitivity comparable to RT-PCR methods.

\section{Results and discussion}

Biofunctionalization. Nanobodies ${ }^{22}$ are compact recognition modules made from the antigen-binding domain (VHH) of an unusual class of heavy-chain-only antibodies found in Camelids ${ }^{23}$. Although several therapeutic ${ }^{24}$ and diagnostic applications ${ }^{25}$ have been developed, nanobodies have not yet been combined with OECT technology. One report described the functionalization of a conventional field effect transistor (FET) with nanobodies. This sensor relied on random chemical immobilization of nanobodies on carbon nanotubes and reached a detection limit of $\sim 1 \mathrm{pM}$ for green fluorescent protein (GFP) ${ }^{26}$. To improve on these previous studies, we capitalized on the fact that nanobodies (and modified versions thereof) can be efficiently expressed in Escherichia coli. We designed a recombinant protein in which the well-characterized anti-GFP nanobody ${ }^{27}$ is fused, through a flexible peptide linker, to a SpyCatcher domain (Fig. 2a). This SpyCatcher domain is specifically recognized by a short SpyTag peptide, triggering the autocatalytic formation of a covalent isopeptide bond linking both of them with very high stability. Originally engineered from a bacterial adhesion protein ${ }^{28}$, the SpyTag/SpyCatcher protein conjugation system has been used for several application ${ }^{29}$ but has not yet been applied to FET or OECT biosensors. The only related application of a nanobody-SpyTag fusion was a passive, impedance-based sensor for the detection of microalga $e^{30}$, which relied on the random chemical immobilization of separated SpyCatcher proteins as a capture reagent.

For our sensor, we wanted to avoid steps where the proteins (nanobody or SpyCatcher) are chemically modified or immobilized in a way that could partially impair their function. Instead, we opted for the immobilization of a chemically modified SpyTag, obtained through regular commercial peptide synthesis, on a 1,6-hexanedithiol (HDT) self-assembled monolayer (SAM), thus forming a combined chemical SAM (chem-SAM) on top of the gold gate electrode. The anti-GFP nanobody ${ }^{27}-$ SpyCatcher fusion protein was genetically encoded, gene synthesized and produced in E. coli. The purified protein construct was then incubated under physiological conditions with the chem-SAM, thus completing the sensing surface with a self-assembled biological SAM (bio-SAM). 

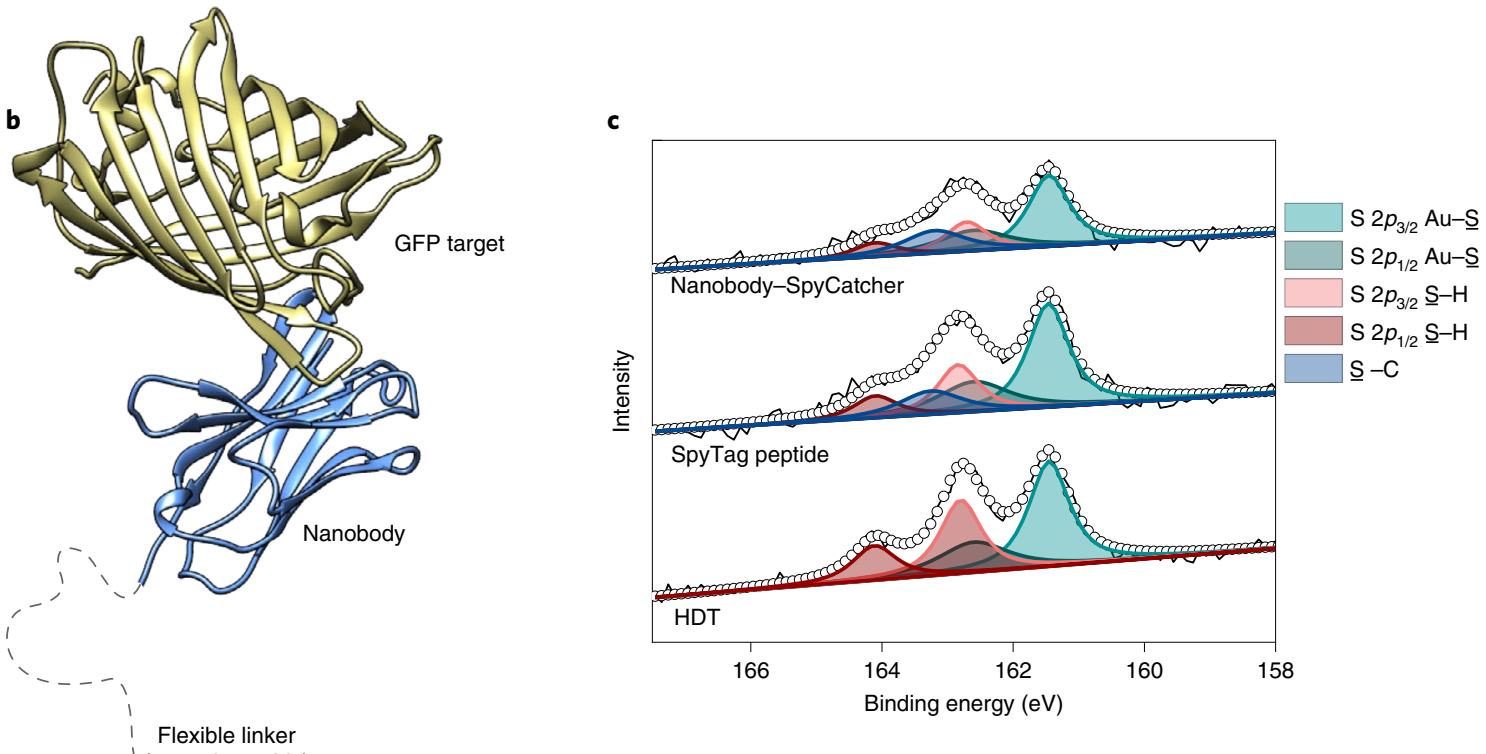
(31 amino acids)
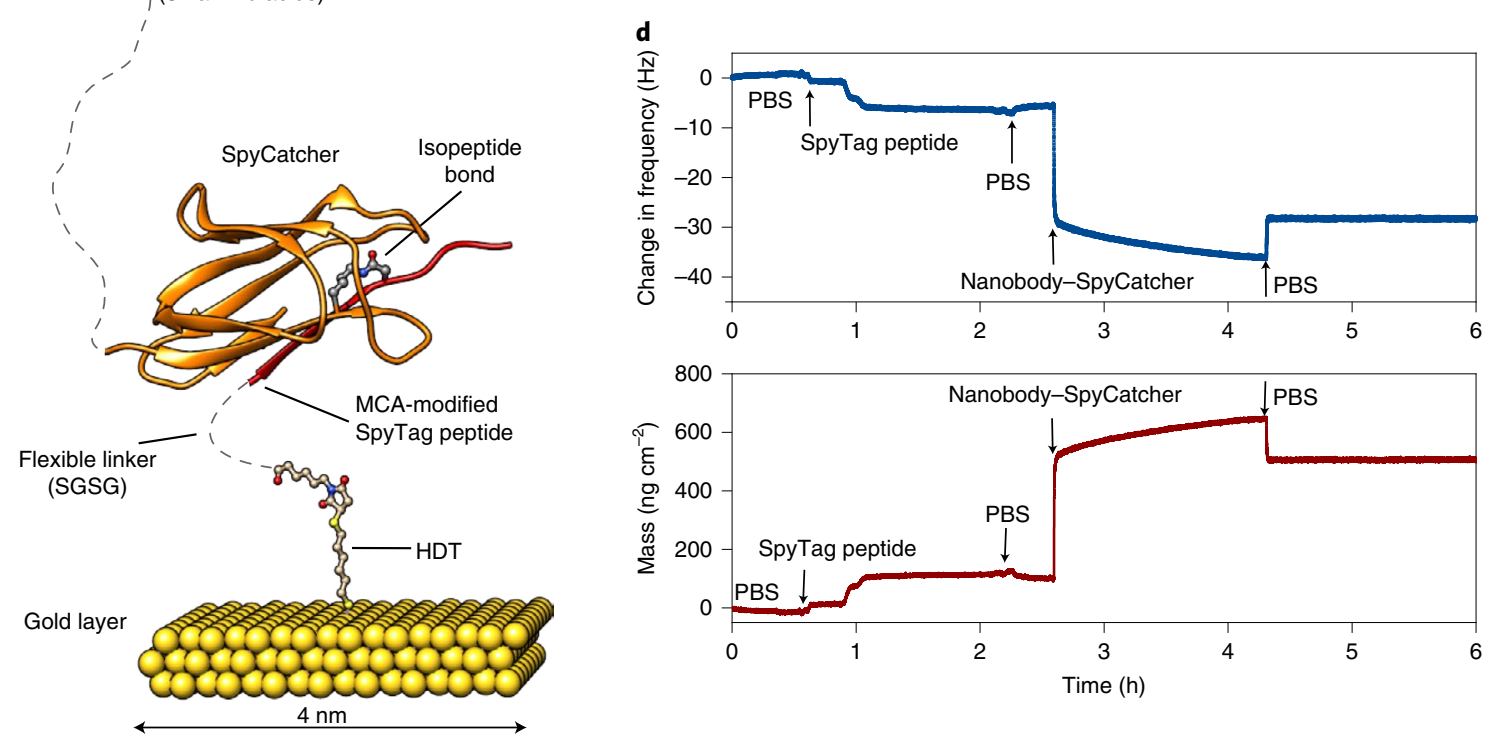

Fig. 2 | Design and characterization of the biofunctionalized gold electrode. a, Annotated sequence of the recombinant fusion protein combining a nanobody recognition module (blue) with the SpyCatcher domain (brown), with an intervening flexible linker (dashed line). b, Structural model of the complete biorecognition layer assembled from HDT and SpyTag (chem-SAM) and nanobody-SpyCatcher fusion protein (bio-SAM) (based on PDB structures $4 \mathrm{MLI}$ and $3 \mathrm{OBO}$ ). Unstructured, flexible linker regions are indicated by dashed lines drawn to scale. c, High-resolution S $2 p$ XPS spectra of the gold electrode recorded after immobilization of the HDT layer, SpyTag peptide and nanobody-SpyCatcher protein (BSA added to the biofunctionalization solution). Spectra for the three functionalization steps are shifted vertically for clarity. d, QCM-D profile for the coupling of the SpyTag peptide and nanobody-SpyCatcher fusion protein (without BSA) in real time, starting from an HDT-coated gold electrode.

This strategy leads to a defined biofunctionalization layer in a precisely controlled molecular configuration and orientation, which can be modeled at atomic resolution (Fig. 2b). A flexible eight-amino-acid glycine-serine linker separates nanobody and SpyCatcher domains dynamically and sterically. Taking into account unstructured residues contributed by the nanobody and SpyCatcher domains, the 4-nm-long nanobody domain is probably separated from the SpyCatcher adapter by about 30 amino acids, forming a $\sim 12$-nm-long flexible molecular leash (Fig. 2a,b). Additional flexibility is introduced by short glycine-serine and alkane linkers that separate the chemically synthesized SpyTag sequence from the underlying HDT, thus also giving the SpyCatcher domain some freedom to move and rearrange itself above the chem-SAM.

The three immobilization steps (HDT, SpyTag peptide and nanobody-SpyCatcher) were monitored through X-ray photoelectron spectroscopy (XPS), quartz crystal microbalance with dissipation (QCM-D), cyclic voltammetry and electrochemical impedance spectroscopy (EIS). Upon functionalization with the HDT layer, the gold electrode displayed characteristic thiol-gold $(\mathrm{S}-\mathrm{Au})$ and free thiol $(-\mathrm{SH})$ peaks in its high-resolution $\mathrm{S} 2 p$ XPS 
spectrum $^{31,32}$, indicating the upright orientation of HDT linked to gold through only one of the two - $\mathrm{SH}$ moieties (with the other terminal pointing away from the surface) (Fig. $2 \mathrm{c}$ and Supplementary Table 1). As we successively introduced SpyTag peptide and then the nanobody fusion protein on top of the chem-SAM, a new peak appeared at about $163.2 \mathrm{eV}$, which we attributed to the $\mathrm{S}-\mathrm{C}$ bond from protein methionine residues (Fig. 2c) 3 $^{33}$. High-resolution $\mathrm{C}$ $1 s$ and $\mathrm{N} 1 s$ XPS spectra further demonstrated the bio-SAM formation by revealing $\mathrm{C}-\mathrm{O}, \mathrm{C}-\mathrm{N}$, and $\mathrm{C}-\mathrm{OOR}$ bonds and nitrogen groups on the surface (Supplementary Fig. 1 and Supplementary Discussion 1). The formation of all layers was further corroborated by EIS and cyclic voltammetry measurements, which showed a decrease in the electrochemical capacitance of the gold electrode and an increase in its charge transfer resistance with the addition of (insulating) chem-SAM and bio-SAM (Supplementary Fig. 2 and Supplementary Discussion 2).

To assess the packing density of biomolecules on the surface, we monitored by QCM-D the immobilization of (1) the maleimide-modified SpyTag peptide and (2) the nanobodySpyCatcher fusion protein on the chem-SAM. We quantified the masses gained from the two conjugates to be 114 and $406 \mathrm{ng} \mathrm{cm}^{-2}$, respectively (Fig. 2d, Supplementary Fig. 3 and Supplementary Discussion 3). Based on molecular weights of 1.76 and $28.4 \mathrm{kDa}$ for the SpyTag peptide and nanobody-SpyCatcher protein, respectively, $39 \times 10^{12}$ SpyTag peptides and $8.6 \times 10^{12}$ nanobody-SpyCatcher molecules were coupled per $\mathrm{cm}^{2}$. This density translates to a mean inter-particle distance of only $3.4 \mathrm{~nm}$ between the SpyCatcher domains at the base of the bio-SAM. Given the size of this domain $(\sim 4 \mathrm{~nm} \times 2.5 \mathrm{~nm})$, we are thus approaching the maximum coupling density that is physically feasible. The formation of this exceptionally high-density biorecognition layer is probably further facilitated by the compact nanobody, which has similar dimensions to the SpyCatcher domain but is given additional freedom to pack and reposition itself through the flexible inter-domain linker (Fig. 2b).

Optimization of protein detection. We used the detection of GFP with the GFP nanobody-conjugated gate electrode as a model to optimize multiple parameters of the sensor design. Initial optimization rounds addressed unspecific binding, which was eliminated by adding bovine serum albumin (BSA) and a mild detergent (Tween-20) to both the nanobody-SpyCatcher protein immobilization and the target-binding solutions (Supplementary Discussion 3). Comparing QCM-D binding traces with (Supplementary Fig. 3) and without BSA (Fig. 2d), we suggest that BSA may be primarily capturing contaminating proteins in solution rather than binding to and blocking the surface in a classical sense. This view is supported by QCM-D traces recorded with another nanobody construct (Supplementary Fig. 4), and by the absence of fluorescently labeled BSA on the gate electrode surface (Supplementary Fig. 5). Nevertheless, the inclusion of BSA in the functionalization protocol was vital for the sensor performance as the addition of BSA to SpyCatcher protein and to the target-binding buffer outperformed other blocking strategies (Supplementary Fig. 6). Next, we optimized the OECT geometry through systematic variation of gate and channel sizes. The highest sensitivity towards GFP was attained with an OECT with a gate electrode area of $0.64 \mathrm{~mm}^{2}$, a channel width of $100 \mu \mathrm{m}$ and a length of $10 \mu \mathrm{m}$ (Supplementary Fig. 7).

The optimized poly(3,4-ethylenedioxythiophene) doped with poly(styrene sulfonate) (PEDOT:PSS) OECTs were operated in phosphate-buffered saline (PBS) under ambient conditions by varying the gate voltage $\left(V_{\mathrm{G}}\right)$ between -0.6 and $0.6 \mathrm{~V}$ while the drain voltage $\left(V_{\mathrm{D}}\right)$ was swept from 0 to $-0.6 \mathrm{~V}$. We assessed the sensing signal by monitoring the drain current $\left(I_{\mathrm{D}}\right)$ as a function of $V_{\mathrm{G}}$ at a fixed $V_{\mathrm{D}}=-0.6 \mathrm{~V}$. The reference (blank) response of the sensor was acquired by exposing the nanobody-functionalized gate electrode to PBS (Fig. 3b). The same electrode was then incubated for $10 \mathrm{~min}$ with a 5- $\mu$ l drop of binding buffer containing GFP, rinsed twice in PBS and then stacked vertically on top of the channel separated by PBS ( $\mathrm{pH} 7.4$; ionic strength $=0.162 \mathrm{M}$ ) to complete the OECT biosensor (Fig. 1a). See Supplementary Fig. 8 for a comparison with a longer incubation time. After gate exposure to GFP, $I_{\mathrm{D}}$ underwent a notable decrease at all of the gate voltages applied. As the GFP concentration increased, this sensor response became more evident (Fig. 3b). Similarly, the transconductance of the devices decreased as GFP interacted with the gate electrode (Supplementary Fig. 9a). In contrast, the device characteristics did not change if the gate electrode was incubated in solutions containing equivalent molar concentrations of non-target molecules such as an FRB-mCherry fusion (Fig. $3 \mathrm{c}$ and Supplementary Fig. 9b) and lysozyme (Supplementary Fig. 10). Lysozyme is abundant in saliva, which we envisioned as a potential biological medium for our SARS-CoV-2 application. mCherry is a red fluorescent protein with low sequence homology but very high structural similarity to GFP and thus challenged the sensor specificity.

The trend in $I_{\mathrm{D}}$ as well as $g_{\mathrm{m}}$ can be clearly attributed to the specific binding of GFP to the nanobody-functionalized gate electrode. Fluorescence images confirmed the specific capture of GFP but not mCherry (Supplementary Fig. 11). The interaction between the nanobody and the GFP increases the impedance at the gate electrode/electrolyte interface (Supplementary Fig. 12), resulting in an increase in the potential decrease therein, which leads to a less efficient capacitive coupling between the gate and the channel (Supplementary Discussion 4). Hence, we measure a reduction in the current and its modulation as GFP binding weakens the electrical driving force acting on the ions. The OECT here provides voltage-to-current transduction and acts as a transconductance amplifier. Torsi and co-workers ${ }^{20}$ observed similar changes in PEDOT:PSS OECT characteristics, where the $I_{\mathrm{D}}$ decreased upon a protein binding event at the functionalized gate electrode. We note that the decrease in $I_{\mathrm{D}}$ is not due to a degradation of the channel over consecutive $I-V$ cycles (Supplementary Fig. 13). Neither did we observe a particular change in the gate current $\left(I_{G}\right)$ upon GFP binding, and the value of $I_{\mathrm{G}}$ was six orders of magnitude lower than the on current of the OECT (Supplementary Fig. 14). Within the gate voltage range that we operate the devices in, no Faradaic reactions that generate current occur at the gate electrode upon analyte binding.

To quantify the sensor response and minimize device-to-device variations, we calculated the normalized response of the OECT by normalizing the target protein-induced change in $I_{\mathrm{D}}$ at a single $V_{\mathrm{D}}$ and $V_{\mathrm{G}}$ to its value previously measured after exposure to the blank solution. The normalized response versus GFP calibration curve revealed that the nanobody-functionalized OECT detects GFP with a lowest limit of detection (LOD) of $23 \mathrm{fM}$ (Fig. 3d). The normalized response was $<5 \%$ even for the highest concentrations of negative controls (mCherry and lysozyme), while it exceeded 30\% for higher (nanomolar) GFP concentrations. Overall, PEDOT:PSS-based OECT biosensors were sensitive to the presence of $6 \times 10^{4} \mathrm{GFP}$ molecules in $5 \mu \mathrm{l}$ of buffer and responded within a dynamic range of six orders of magnitude. This sensitivity is on par with commercial colorimetric enzyme-linked immunosorbent assays, which take hours to complete, require larger sample volumes and have a far narrower dynamic range ${ }^{34}$.

Sensing with accumulation-mode OECTs. OECTs can be constructed for either depletion- or accumulation-mode operation. Currently, the most often used channel material is PEDOT:PSS; this is intrinsically doped and therefore conductive by default. PEDOT:PSS OECTs thus operate in depletion-mode. Application of a positive gate voltage $V_{\mathrm{G}}$ injects cations into the channel, which compensate for the depleted holes and switch the transistor off. For continuous and long-term use, the depletion-mode operation 
<smiles></smiles>

e<smiles>CCOCCOc1cc(C(C)(C)C)sc1-c1sc(-c2cc(OC)c(-c3sc(C(C)(C)C)cc3OC)s2)cc1OCCOC(C)(C)C</smiles>

b
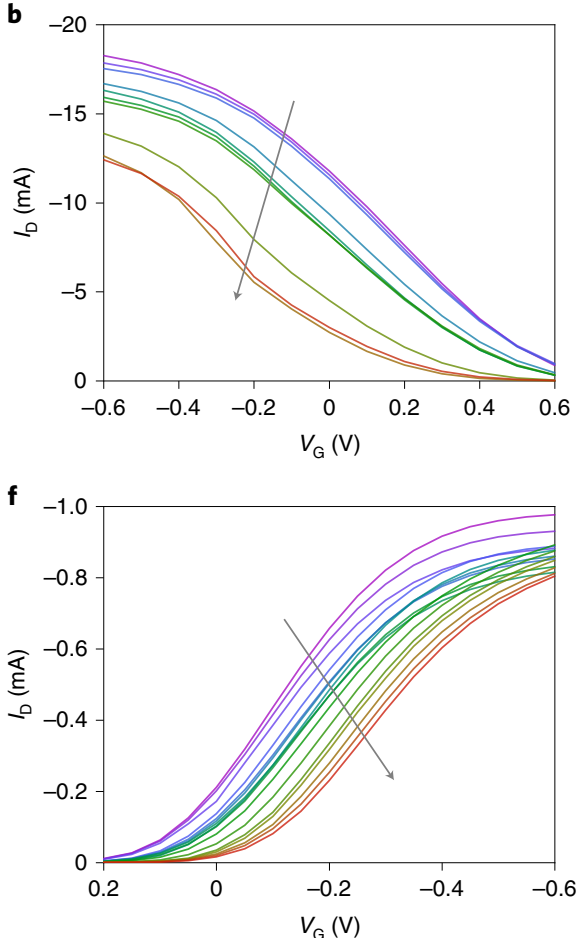

c

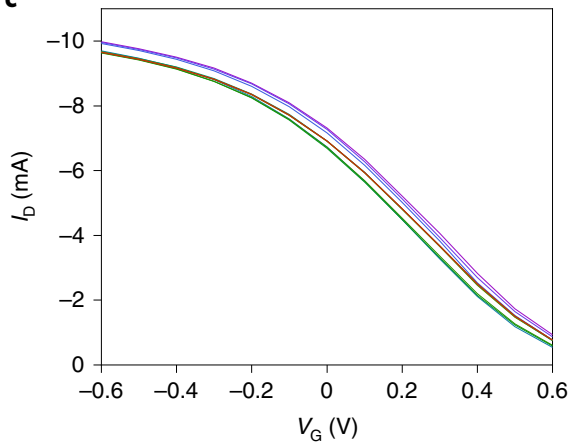

g

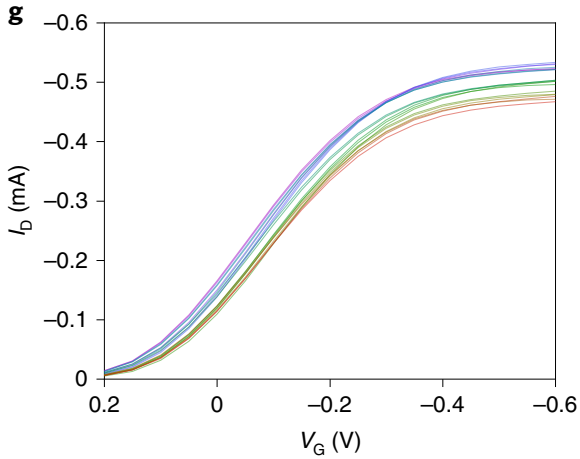



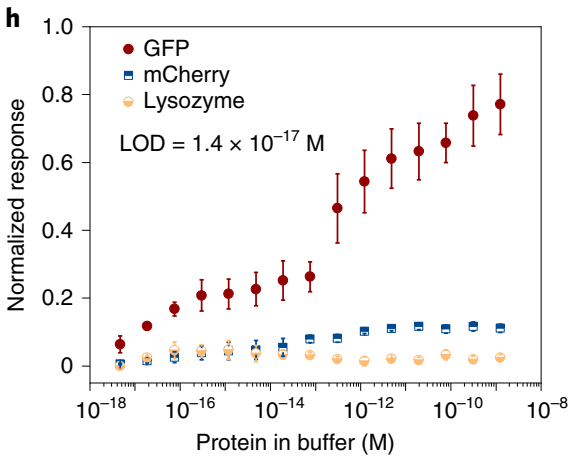

Fig. 3 | Performance of GFP nanobody-functionalized OECT biosensors. a, Chemical structure of the PEDOT:PSS channel material. b,c, Steady-state characteristics of the depletion-mode PEDOT:PSS OECT gated with a GFP nanobody-functionalized electrode are shown when exposed to its target GFP (b) or its non-target mCherry (c). The arrow indicates increasing protein concentration. $\mathbf{d}$, Resulting normalized response of the sensor to GFP, mCherry and lysozyme. e, Chemical structure of the novel p(g0T2-g6T2) channel material. f,g, Steady-state characteristics of the accumulation-mode OECT after exposure to GFP target (f) or non-target mCherry ( $\mathbf{g}$ ). The arrow indicates increasing protein concentration. $\mathbf{h}$, Normalized response to GFP, mCherry and lysozyme. The normalized response was calculated as normalized response $=\left(\left|I_{\mathrm{D}}-I_{0}\right|\right) / I_{0}$ (the current change normalized to the baseline signal). The average value of the normalized response is plotted. The error bars in $\mathbf{d}$ and $\mathbf{h}$ represent the standard deviation calculated from at least three gate electrodes. Note that the PEDOT:PSS channel of the depletion-mode OECT is initially on (that is, high $I_{D}$ ) and switches off (that is, low $I_{D}$ ) upon the injection of cations $\left(V_{G}\right.$ sweeping from -0.6 to $\left.+0.6 \mathrm{~V}\right)$. Conversely, the $\mathrm{p}(\mathrm{gOT} 2-\mathrm{g} 6 \mathrm{~T} 2)$ channel of the accumulation-mode OECT is initially off and switches on upon the injection of anions $\left(V_{G}\right.$ sweeping from +0.2 to $\left.-0.6 \mathrm{~V}\right)$. In both cases, analyte binding reduces the capacitive coupling between the gate and the channel and lowers the gating efficiency and hence the magnitude of $I_{D}$.

may negatively affect material stability and power consumption as it implies high operating currents and relatively high $V_{\mathrm{G}}$ to run the device. Recent studies expanded the available OECT channel materials through the chemical design of undoped, conjugated backbones, which were functionalized with hydrophilic side chains that facilitate ion injection and transport in the film ${ }^{16,35}$. OECTs comprising these materials operate in accumulation-mode (that is, the channel is initially off and generates a current only upon application of a small gate voltage ${ }^{17}$. This operation mode allows for low-power electronics applications, improves device stability and is more compatible with integrated circuit designs that conventionally involve accumulation-mode transistors. Nevertheless, accumulation-mode OECTs for biochemical sensing have not yet been reported. We therefore replaced PEDOT:PSS with a recently designed conjugated polymer, p(g0T2-g6T2) ${ }^{36}$ (Fig. 3e). p(g0T2-g6T2) is a mixed (ionic and electronic) conductor. A negative $V_{\mathrm{G}}$ pushes anions into the film that compensate for the holes injected from the metal contacts, thus turning the device on (Supplementary Fig. 15). The maximum $g_{\mathrm{m}}(15 \mathrm{mS})$ occurs at a $V_{\mathrm{G}}$ of around $-0.3 \mathrm{~V}$ (Supplementary Fig. $15 \mathrm{~b})$. The transconductance efficiency $\left(g_{\mathrm{m}}\right.$ obtained per unit current) is very high at low $V_{\mathrm{G}}$ (with the maximum slope of $60 \mathrm{mV} \mathrm{dec}^{-1}$ at $V_{\mathrm{G}} \approx 0.15 \mathrm{~V}$; Supplementary Fig. $15 \mathrm{c}$ ) due to the exponential $I_{\mathrm{D}}$ versus $V_{\mathrm{G}}$ characteristics. This behavior is similar to the subthreshold region of operation in traditional inorganic metal oxide semiconductor $\mathrm{FETs}^{37}$. The material also shows exceptional operational and environmental stability (Supplementary Fig. 16), thus overcoming the limitations of the currently available organic mixed (semi) conductors $^{36}$. 
Figure $3 \mathrm{f}$ shows the typical transfer characteristics of $\mathrm{p}$ (g0T2-g6T2) OECTs gated with the nanobody-functionalized gold electrodes incubated in solutions with varying GFP concentrations. As GFP bound to the gate, $I_{\mathrm{D}}$ decreased for all gate voltages. This decrease was accompanied by a significant shift in the threshold voltage $\left(V_{\mathrm{th}}\right)$ towards more negative values (Supplementary Fig. 17). Similar to PEDOT:PSS sensors, p(g0T2-g6T2) OECTs showed no significant response to mCherry (Fig. 3g) and lysozyme (Supplementary Fig. 18) (at closer inspection, our data suggest a minor cross-reaction of the anti-GFP nanobody with the structurally similar mCherry). The sensor responded to a GFP concentration as low as $4.7 \mathrm{aM}$ (normalized response $=7 \%$ ) (Fig. $3 \mathrm{~h}$ ) with a dynamic range spanning ten orders of magnitude. We calculated the lowest LOD for these devices to be $14 \mathrm{aM}$, corresponding to 42 molecules in the $5 \mu \mathrm{l}$ sample. Compared with the otherwise identical PEDOT:PSS-based sensor, the accumulation-mode OECT thus showed a 1000-fold improved sensitivity (aM compared with fM) and yet a larger dynamic range (ten orders of magnitude, from aM to $\mathrm{nM}$, compared with six orders of magnitude). Moreover, we noted a much lower operating voltage. The target response was maximized (and the off-target response was minimized) at $V_{\mathrm{G}}=V_{\mathrm{D}}=-0.1 \mathrm{~V}$, compared with $V_{\mathrm{G}}=V_{\mathrm{D}}=-0.6 \mathrm{~V}$ for the PEDOT:PSS OECT (Supplementary Fig. 19). Such characteristics would, in principle, allow for nanobody-OECT reader devices with a very compact build and long battery life.

The ability to detect the binding of analytes to only a few out of 55 billion receptors on our gate electrode surface seems intriguing. Monitoring of single-molecule binding to large gate electrodes was previously demonstrated in an electrolyte-gated organic FET recognizing immunoglobulin $\mathrm{G}$ as the target ${ }^{13}$. Macchia and colleagues ${ }^{14}$ attributed single-molecule detection to a combination of: (1) the 1000 -fold amplification of input to output signal provided by their transistor; and (2) the perturbation of a tight hydrogen-bonding network in their chem-SAM, which they suggested to propagate as a defect over larger areas of the electrode ${ }^{13,38}$. In contrast, our sensor does not have a hydrogen-bonding network in or near the chem-SAM. However, the OECTs developed in this work have higher gains (the $g_{\mathrm{m}}$ is on the order of $\mathrm{mS}$ rather than $\mu \mathrm{S}$ of typical electrolyte-gated organic FETs), permitting the amplification of input signals by a factor of one million. Indeed, without the amplification endowed by the OECT circuitry, we cannot resolve gate capacitance changes at such low concentrations (Supplementary Fig. 12a,b). A further improvement over previous designs is the very dense coupling of compact and intact receptor units in a, presumably, near-optimal binding orientation. Assuming the domain is diffusing within a $20-\mathrm{nm}$ layer above the sensor surface (Fig. 2b), we obtain a local nanobody concentration of $\sim 700 \mu \mathrm{M}$, which is far above the dissociation constant. Each target molecule diffusing into this capture layer will therefore necessarily bind (Supplementary Discussion 5). Once bound, the analyte is extremely unlikely to escape the sensor surface owing to a low $k_{\text {off }} \operatorname{rate}^{39}\left(\sim 1.5 \times 10^{-4} \mathrm{~s}^{-1}\right.$, corresponding to an 80 -min half-life, which is unmeasurable according to our own surface plasmon resonance (SPR) measurements; Supplementary Fig. 20). The likelihood of analyte escape is further reduced by a high possibility of re-binding within the high-density receptor phase ${ }^{40}$. Our gate electrode thus operates as a kinetic trap for target molecules, while unspecific contaminants are washed away due to their faster off rates. Analyte diffusion from the binding volume to the sensor surface ${ }^{40}$ is probably a major bottleneck $^{41}$, which we address through a large gate surface ${ }^{42}$ and intermittent mixing during incubation. Moreover, the high receptor density makes unproductive collisions between analyte and surface very unlikely. The large gate area and high receptor density also combine to a high binding capacity (corresponding to an analyte concentration of $18 \mathrm{nM}$ in the $5 \mu \mathrm{l}$ solution volume), which increases the sensor's dynamic range. In summary, sensor performance is thus driven by the high transconductance, the large binding electrode surface and a very high receptor coupling density and quality.

Detection of SARS-CoV-2 and Middle East respiratory syndrome coronavirus (MERS-CoV) antigens. The VHH72 nanobody was originally raised against the SARS-CoV receptor-binding domain (RBD). Subsequently, VHH72 was shown to also bind SARS-CoV-2 at a slightly reduced affinity, and it became the first publicly available nanobody sequence against this new target ${ }^{43}$. Our VHH72SpyCatcher fusion construct expressed well in E. coli and could be purified to a high yield ( $54 \mathrm{mgl}^{-1}$ culture) as a monomeric protein. Although SARS-CoV-2 was our primary diagnostic target, we also designed and expressed constructs for the detection of MERS-CoV based on the previously reported nanobodies VHH83 (ref. ${ }^{44}$ ), VHH04 (ref. ${ }^{44}$ ) and VHH55 (ref. ${ }^{43}$ ). The VHH04-SpyCatcher fusion yielded high-quality monomeric protein and was advanced to OECT experiments. Both VHH72 and VHH04 bind to the RBD of the homotrimeric (SARS or MERS, respectively) coronavirus spike protein $^{45}$, which protrudes from the virus surface in multiple (about 100$)$ copies. The RBD is a small $(27-\mathrm{kDa})$ protein domain within the larger $\mathrm{S} 1$ subunit $(76 \mathrm{kDa})$ and is directly responsible for recognition and binding of the specific host cell receptor. Detection of RBD would therefore be a good indicator for the presence of infectious viral particles. SPR experiments confirmed the binding of both nanobody fusion proteins with their respective, recombinantly expressed SARS-CoV, SARS-CoV-2 or MERS-CoV target proteins (Supplementary Fig. 21). As expected, VHH04 showed the highest affinity $\left(K_{\mathrm{D}}=0.1 \mathrm{nM}\right)$ and slowest $k_{\text {off }}\left(2 \times 10^{-4} \mathrm{~s}^{-1}\right)$ for its target MERS-CoV S1. VHH72 bound its primary target SARS-CoV(1) RBD with lower affinity $\left(K_{\mathrm{D}}=7 \mathrm{nM}\right)$, owing to a faster dissociation $\left(3 \times 10^{-3} \mathrm{~s}^{-1}\right)$. The interaction with the closely related RBD of SARS-CoV-2 showed a mixed signal, where a smaller share of fast-on, fast-off binding events was distorting the main slow-on, slow-off binding regimen $\left(K_{\mathrm{D}}=23 \mathrm{nM} ; k_{\text {off }}=3 \times 10^{-4} \mathrm{~s}^{-1}\right)$. Such a signal was also reported for the interaction with a more recently developed nanobody ${ }^{46}$ and may indicate an intrinsic heterogeneity of this RBD.

Nevertheless, SARS-CoV nanobody-functionalized OECTs showed an excellent response to the SARS-CoV-2 RBD and S1 subunit, regardless of the channel material used (Fig. 4a,b). Incubation of the same gate electrodes with various concentrations of GFP did not evoke any current response, demonstrating the high specificity of the VHH72-functionalized gate for the viral proteins. The device also responded to the original target of VHH72-the SARS-CoV-1 RBD (Supplementary Fig. 22). In a direct comparison, the higher affinity of VHH72 for the SARS-CoV-1 RBD compared with the SARS-CoV-2 RBD only translated into a small signal increase. In contrast, the larger SARS-CoV-2 S1 spike protein (comprising the same RBD) generated much larger current changes than the isolated RBD. This increase in the normalized response may stem from higher affinity or size or both. We assume that the S1 subunit will, at least partially, form trimers in solution (all concentrations given in this study refer to the monomer) and thus benefit from avidity effects with substantially increased affinity (and prolonged residence) on the gate surface. Compared with the RBD alone, either the S1 monomer or the trimer would also cover a larger area on the functionalized surface than the isolated RBD. Akin to our GFP sensors, p(g0T2-g6T2) OECTs showed higher sensitivities than PEDOT:PSS devices. p(g0T2-g6T2) OECTs detected SARS-CoV-2 S1 at $4.7 \mathrm{aM}$ with a $30 \%$ change in the normalized response (s.d. $=7 \%$ at most), translating to a nominal LOD of $18 \mathrm{zM}$. In fact, a single-molecule in our $5 \mu \mathrm{l}$ measurement volume corresponded to a concentration of $0.33 \mathrm{aM}$, which appeared to be easily detected.

Having confirmed the single-molecule sensitivity and high specificity of SARS-CoV nanobody-OECTs when target proteins were captured in the binding buffer, we turned our attention to 

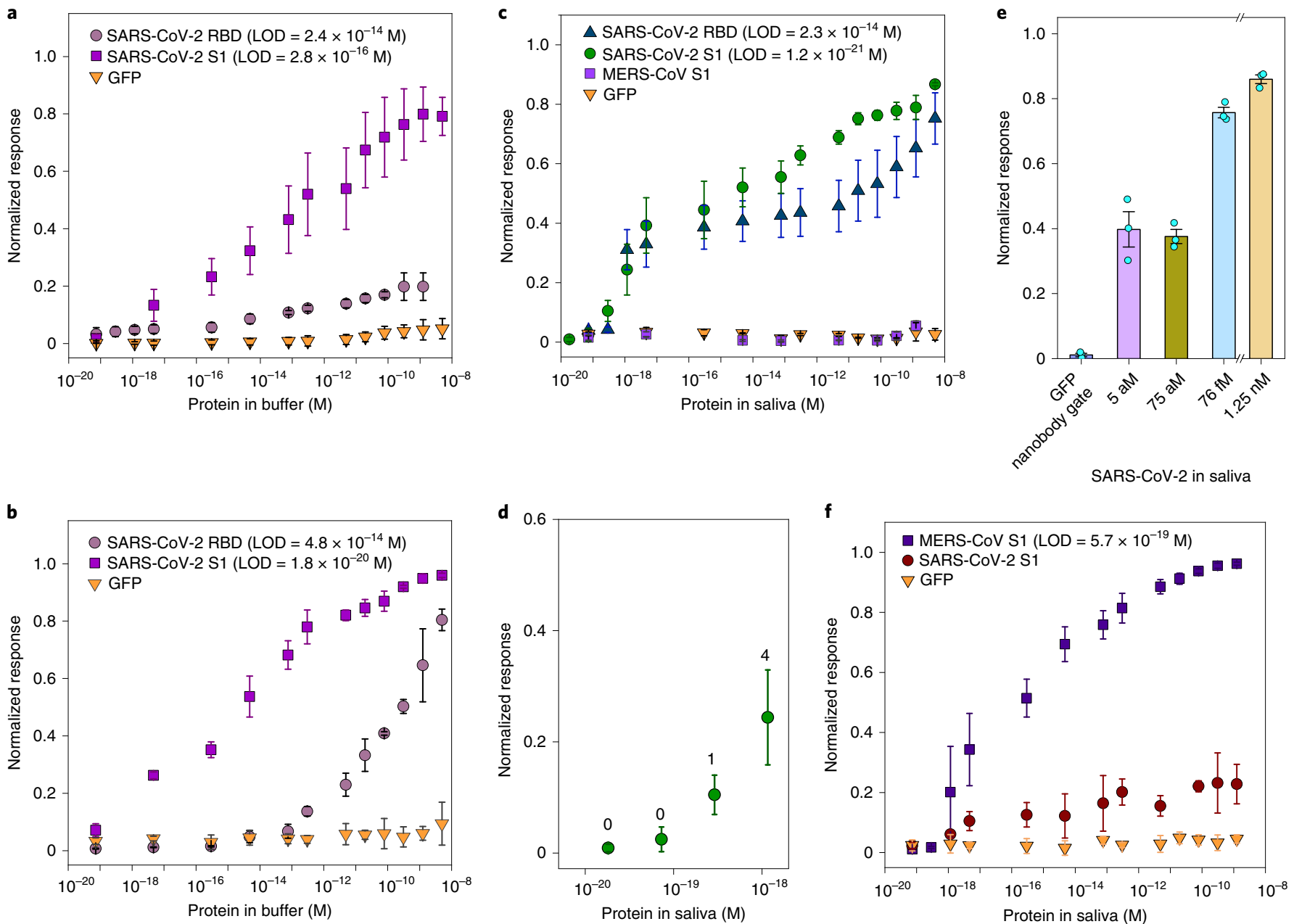

Fig. 4 | Performance of the SARS-CoV and MERS nanobody-functionalized OECTs in detecting SARS-CoV-2 or MERS-CoV proteins. a,b, Normalized response of SARS-CoV nanobody-functionalized OECTs to SARS-CoV-2 RBD or S1 and GFP for channels comprising PEDOT:PSS (a) and p(g0T2-g6T2) (b). c, Response of SARS-CoV nanobody-functionalized OECTs comprising p(gOT2-g6T2) channels to SARS-CoV-2 proteins, GFP and MERS-CoV S1 spiked into human saliva. d, Normalized response of SARS-CoV-2 sensors at single-molecule concentrations. The theoretical number of spike protein molecules within the $5 \mu \mathrm{l}$ sample is indicated above each data point. e, Normalized response of SARS-CoV-2 sensors to randomly selected saliva samples containing various amounts of SARS-CoV-2 S1. One of the samples ( $1.25 \mathrm{nM}$ target protein) was measured with the GFP nanobody gate as a negative control. In a-e, the blank measurements were obtained after incubation in PBS. The average value of the normalized response is plotted. The error bars show the s.e.m. from three gate electrodes measured on one channel. f, Response of MERS nanobody-functionalized OECTs comprising p(g0T2-g6T2) channels to MERS-CoV S1, SARS-CoV-2 S1 and GFP spiked into human saliva. The average normalized response is shown, along with error bars representing the s.d. calculated from measurements with at least three gate electrodes. The normalized response was calculated as normalized response $=\left(\left|I_{D}-I_{0}\right|\right) / I_{0}$ (the current change normalized to the baseline signal).

sensor performance in saliva. For RT-PCR detection of SARS-CoV-2, saliva samples match nasal swabs in sensitivity and specificity ${ }^{47-49}$ and are clearly the better medium for POC or field applications. We therefore challenged SARS-CoV nanobody-functionalized OECTs comprising p(g0T2-g6T2) channels with human saliva samples into which we spiked predetermined amounts of target and non-target proteins. The normalized response increased with the concentration of SARS-CoV-2 proteins in the saliva and showed only negligible change when the same gate electrode was exposed to GFP (Fig. 4c). As before, the sensor response already picked up at the single-molecule concentration threshold (Fig. 4d). The sensitivity for SARS-CoV-2 S1 in saliva $\left(\mathrm{LOD}=1.2 \times 10^{-21} \mathrm{M}\right)$ was comparable to that in buffer $\left(\mathrm{LOD}=1.8 \times 10^{-20} \mathrm{M}\right)$, indicating that the complex composition of saliva did not hamper the association between the nanobody and its target. The OECT could also detect SARS-CoV-2 S1 mixed into human serum (Supplementary Fig. 23), indicating its potential use for a wide range of other diagnostic applications.
So far, we have characterized sensor responses through the repeated exposure of one gate electrode to increasing target concentrations (all of the results are averages over several electrodes gating several channels each). To better emulate real-world screening conditions, we directly exposed individual gate electrodes to a single random concentration of SARS-CoV-2 S1 in saliva (Fig. 4e). The normalized response values varied accordingly and corresponded to the values obtained in the regular dose curves. In contrast, even high concentrations of SARS-CoV-2 S1 did not evoke any response on a gate functionalized with GFP nanobody. Nevertheless, the determination of exact concentration values is currently hindered by variation between individual gate electrodes and channels, which is exacerbated by stochastic variations in molecule numbers at very low concentrations. The manual analyte incubation and mixing may cause additional error. When moving towards practical applications, accurate concentration measurements will probably require a more streamlined and automated fabrication and operation of the device. 

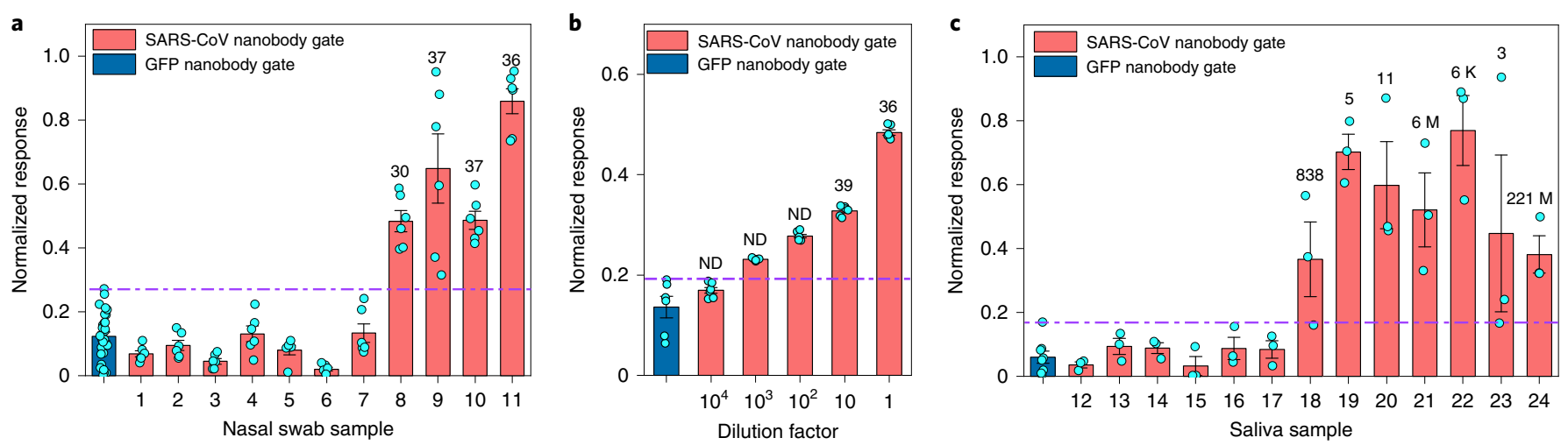

Fig. 5 | SARS-CoV-2 detection in clinical samples. a, Normalized sensor response for nasopharyngeal swab samples $(n=11)$ from healthy volunteers (1-7) and COVID-19-positive samples from walk-in patients (8-11). Cycle threshold values from RT-PCR are indicated above the positive samples. $\mathbf{b}$, Sensor response for a tenfold dilution series of nasal swab sample number 8 , along with RT-PCR results after RNA extraction from the same diluted samples (values above the bars). ND, not detected. The error bars in $\mathbf{a}$ and $\mathbf{b}$ indicate the s.d. measured from two gate electrodes combined with three channels. All positive samples were also measured using two GFP nanobody gate electrodes each. $\mathbf{c}$, Average sensor response for saliva samples $(n=13)$ taken from healthy volunteers (12-17) and outpatients who had recently tested positive for COVID-19 (18-24). RT-qPCR was performed on each sample and the viral copy numbers in the $5 \mu \mathrm{l}$ sample volume are indicated above the bars. The error bars show the s.e.m. from three gate electrodes measured on one channel. Measurements with one GFP nanobody gate per COVID-19-positive sample served as an additional negative control. As before, the normalized response was calculated as normalized response $=\left(\left|I_{D}-I_{0}\right|\right) / I_{0}$ (the current change normalized to the baseline signal). $K$, thousand; $M$, million.

MERS-CoV is the only close relative of SARS-CoV-2 in active circulation $^{50}$. The MERS-CoV nanobody-functionalized gate electrode detected single MERS-CoV S1 protein molecules in saliva, with a nominal detection range from $100 \mathrm{zM}$ to $1 \mathrm{nM}$ (Fig. 4f). A weak cross-reaction of VHH04 with SARS-CoV-2 target protein could already be anticipated from SPR measurements (Supplementary Fig. 21). In contrast, despite the high structural similarity between the two proteins, the SARS-CoV sensor did not respond to MERS-CoV S1 (Fig. 4c). Remarkably, the MERS-CoV sensor again achieved single-molecule sensitivity and ten orders of magnitude dynamic range in complex biological media without previous optimization to this particular target (see Supplementary Table 2 for the LODs of all of the sensors used in this study and Supplementary Table 3 for the sensitivity values). Our findings thus demonstrate the sensitivity and selectivity of nanobody-OECTs in complex biological media and at physiologically and clinically relevant protein concentrations. We expect that the nanobody-OECT technology can be rapidly adapted to detect any protein antigen for which nanobodies are available. A comparison of our OECT sensor performance with other similar thin-film transistor-based devices developed for immunosensing indicates the superiority of this sensor technology (for example, in terms of dynamic range, power consumption, miniaturization and compatibility with unprocessed human samples; Supplementary Table 4). OECTs also compare favorably to optical methods for single-molecule detection, such as flow-based immuonassays $^{51}$ and plasmonic nanosensors ${ }^{52}$ : Targets are not limited by size, and the lack of labeling allows for very short sample preparation and device operation times.

Detection of SARS-CoV-2 in clinical samples. We first validated our sensors on human nasopharyngeal swab samples collected from outpatients at a clinical testing facility. Swabs were received in a commercial universal transport medium (UTM; proprietary composition) and analyzed after 2 days. A calibration curve with SARS-CoV-2 S1 protein confirmed sensor operation in this UTM, albeit at lowered sensitivity (the LOD for SARS-CoV-2 S1 in UTM was $1.9 \times 10^{-14} \mathrm{M}$ ) (Supplementary Fig. 24). All four nasal swab samples that had previously been tested as positive were confirmed to be positive by the OECT. Notably, all samples had low viral loads (RT-PCR cycle threshold $=30$ to 37 ; close to the detection limit
$($ cycle threshold $=40)$ ). For a direct comparison of detection limits, we prepared a tenfold dilution series in UTM of one patient sample, mixed each dilution 1:1 with the binding buffer and subjected it side by side to sensor measurements, as well as RNA extraction and RT-PCR (Fig. 5b). The sensor's above-noise response appeared to outperform RT-PCR by one order of magnitude.

In a second validation, saliva was collected from six healthy volunteers and seven volunteers who had recently been confirmed as COVID-19 positive. Each sample was diluted fourfold in a modified, virus-inactivating lysis buffer and subjected to three independent measurements. Positive samples were additionally measured with one GFP nanobody gate electrode each. Quantitative PCR with reverse transcription (RT-qPCR) on each sample (calibrated against $\mathrm{N}$-gene complementary DNA) confirmed the six negatives and revealed a wide range of viral loads from 2,700 to $1.8 \times 10^{12}$ copies per $\mathrm{ml}$ for the others (Supplementary Table 5 and Supplementary Fig. 25). Despite substantial variation in individual sensor readings, average OECT signals reliably agreed with RT-qPCR on the presence or absence of SARS-CoV-2 in all samples (Fig. $5 \mathrm{c}$ and Supplementary Fig. 26). However, in contrast with the previous protein measurements, there was no correlation between sensor signal and the viral loads established by RT-PCR. However, the dilution series of virus material from a nasal swab in commercial UTM shows that a concentration-dependent signal can be achieved (Fig. 5b). We suspect that our sensor response is obscured by the co-recruitment of spike proteins attached to viral debris of largely varying size and composition. Our preliminary experience with patient samples thus suggests a qualitative sensor performance on par with RT-PCR and RT-qPCR, but also points to areas that need to be optimized for its application as a diagnostic device.

\section{Outlook}

We have introduced a label-free electrochemical immunosensing technology with single-molecule sensitivity and a large dynamic range. The nanobody-OECT biosensor detects specific protein molecules from unprocessed samples under ambient conditions after only $10 \mathrm{~min}$ of incubation with the disposable biofunctionalized gate electrode. The sensor can be rapidly adapted to any target for which nanobodies exist or can be raised. After optimizing the sensor for GFP detection, the simple exchange of nanobodies gave 
attomolar sensitivities for SARS-CoV-2 and MERS spike proteins in saliva or buffers without any further modification to the sensor or protocol. Preliminary tests with samples from patients with COVID19 and healthy volunteers demonstrate a sensor accuracy and sensitivity on par with RT-PCR. For COVID-19, the detection of intact spike protein may, in fact, be a better indicator of infectious virus than RT-PCR, as the latter is also detecting membrane-associated viral RNA fragments long after the infection has been cleared ${ }^{53}$.

We attribute the performance of our sensor to several synergistic design choices. The OECT architecture outperforms other transistor technologies in terms of signal amplification and responds to changes far from the sensor surface, despite its simplicity and miniaturized geometry. These characteristics allowed us to tether biorecognition domains through long flexible linkers. A bioconjugation strategy, based on the autocatalytic assembly of a protein adapter with a chemically synthesized peptide, allowed for the oriented coupling of chemically unmodified recognition proteins at an exceptionally high density, which was limited only by the size of the proteins used. The choice of nanobodies over classic antibodies (or antibody fragments) further improved the density and robustness of the biorecognition layer. The resulting three-dimensional and flexible capture layer displayed a kinetically controlled target-binding regimen that is not limited by equilibrium dissociation constants. Moreover, a new solution-processable organic semiconductor enabled the sensor to operate in accumulation-mode, which further improved the sensitivity and stability by providing large changes in the output current for operation at very low-biasing conditions.

Several additional features recommend this sensor architecture for POC applications. The transistor base capable of recording from multiple micrometer-scale channels is reusable, allowing a stable electronic base to be combined with disposable biofunctionalized gate electrodes. Power consumption of the sensor is minimal and compatible with a hand-held battery-driven reader. Test results are currently obtained in less than $15 \mathrm{~min}$, and we expect that changes in our 10-min incubation protocol can further accelerate the procedure. Real-time measurements are technically feasible when the sensor is integrated with microfluidics. Our current laboratory-scale prototype relies on careful handling by skilled and experienced operators. However, the functionalization is simple and modular, and both the gate electrode and the transistor base are easy to fabricate. The device operation consists of four easy steps: blank measurement, incubation, rinsing and sample measurement. Device assembly and packaging will now have to be engineered into, for instance, a single-housing flow-cell format that can be used by minimally trained personnel. The speed, performance and versatility of our nanobody-functionalized OECT, and its compatibility with many sample types, suggest that this biosensor technology can complement or replace a wide range of clinical and non-clinical diagnostic assays.

\section{Methods}

Materials. Sodium chloride, Tween-20, glycerol, HEPES, BSA,

(3-glycidyloxypropyl) trimethoxysilane (GOPS), sodium dodecylbenzenesulfonate, ethylene glycol, HDT, 1,3-propanedithiol, 3-mercaptopropionic acid, albuminfluorescein isothiocyanate conjugated bovine protein, human serum and PBS ( $\mathrm{pH} 7.4)$ were purchased from Sigma-Aldrich and used as received. PEDOT:PSS (PH1000) was received from Heraeus. All aqueous solutions were prepared with ultrapure water (Millipore Milli-Q). p(g0T2-g6T2) was synthesized according to a procedure reported previously ${ }^{36}$. The protein purification materials used were: agar, LB broth, 2x YT broth, kanamycin, glucose, isopropyl $\beta$-D-1-thiogalactopyranoside, BugBuster (Novagen), cOmplete Protease Inhibitor mix (Sigma-Aldrich), Benzonase (Novagen), egg lysozyme (Fluka), Tris(2 carboxyethyl)phosphine, Tris(hydroxymethyl)aminomethane hydrochloride (Tris- $\mathrm{HCl}$ ), imidazole, glycerol, dithiothreitol (DTT), ethylenediaminetetraacetic acid (EDTA), D-desthiobiotin and $10 \mathrm{~K}$ Amicon Ultra spin concentrators (Milipore). The following purification columns and SPR materials were purchased from GE Healthcare: HisTrap HP $(5 \mathrm{ml})$, StrepTrap HP $(5 \mathrm{ml})$, Superdex75 16/600 and Biacore NTA SPR sensor chips (BR100034). The MCA-SpyTag peptide was from GenScript (Peptide Synthesis Service). The following viral target proteins were purchased from Sino
Biological: SARS-CoV RBD (40150-V08B2), SARS-CoV-2 RBD (40592-V08H), SARS-CoV-2 S1 (40591-V08B1) and MERS-CoV S1 (40069-V08H). The Universal Transport Medium kit (UTM proprietary composition) was purchased from Noble Biosciences.

Fabrication of OECTs and the gate electrode. OECTs were microfabricated on glass substrates based on established protocols using standard photolithography and parylene $\mathrm{C}$ peel-off techniques ${ }^{5,55}$. The process starts with the first layer of photoresist (AZ5214) being spin coated and exposed to ultraviolet light using contact aligner to create Au electrodes and interconnection pads. The photoresist patterns were generated with AZ 726 MIF developer, followed by metal sputtering of $10 \mathrm{~nm} \mathrm{Cr}$ and $100 \mathrm{~nm} \mathrm{Au}$ and a standard lift-off process using hot dimethyl sulfoxide. Next, we coated the second layer of photoresist AZ9260 on the substrates and developed them using AZ developer. A parylene $\mathrm{C}$ layer was deposited to insulate the gold interconnects. The OECT channel was patterned by reactive ion etching, using a second layer of parylene $\mathrm{C}$, which was peeled off to yield a length of $10 \mu \mathrm{m}$ and a width of $100 \mu \mathrm{m}$. The aqueous dispersion of PEDOT:PSS containing ethylene glycol ( $5 \mathrm{vol} \%)$, sodium dodecylbenzenesulfonate $(0.25 \mathrm{vol} \%)$ and GOPS ( $1 \mathrm{wt} \%$ ) was sonicated for $30 \mathrm{~min}$ and then spin coated (3,000 r.p.m.; $45 \mathrm{~s}$ ) on the substrates, leading to a film thickness of $\sim 160 \mathrm{~nm}$. The PEDOT:PSS OECTs were annealed at $140^{\circ} \mathrm{C}$ for $1 \mathrm{~h}$ to activate GOPS and avoid dissolution of the polymer film in aqueous medium. p(g0T2-g6T2) films were spin coated (800 r.p.m.; 45 s) from a chloroform solution $\left(5 \mathrm{gl}^{-1}\right)$ on the substrates to yield a film thickness of $\sim 70 \mathrm{~nm}$ in the channel. All devices were rinsed with deionized water before use.

To fabricate the gate electrode, we used 175- $\mu$ m-thick Kapton (polyimide) substrates. The Kapton was first cut with a $\mathrm{CO}_{2}$ laser (Universal Laser Systems; PLS6.75) into a square geometry that defined the sensor active area $(0.8 \times 0.8 \mathrm{~mm})$. We then sonicated the substrates, first in isopropyl alcohol and then in deionized water for $30 \mathrm{~min}$ each. We sputtered $10 \mathrm{~nm}$ chromium or titanium and $100 \mathrm{~nm}$ $\mathrm{Au}$ onto these cleaned substrates. Before the functionalization, the electrodes were electrochemically cleaned in $10 \mathrm{mM}$ sulfuric acid $\left(\mathrm{H}_{2} \mathrm{SO}_{4}\right)$ using cyclic voltammetry. Ten cyclic voltammetry cycles with a potential between -0.2 and $1.2 \mathrm{~V}$ were applied at a scan rate of $100 \mathrm{mV} \mathrm{s}^{-1}$.

Biofunctionalization of gate electrodes. The chem-SAM solution was prepared in absolute ethanol containing $1 \mathrm{mM}$ HDT, as described by others ${ }^{56}$. The Au electrodes were immersed in this solution for $1 \mathrm{~h}^{32}$. The electrodes were rinsed with absolute ethanol and dried under an $\mathrm{N}_{2}$ stream. The electrodes were then incubated for $1 \mathrm{~h}$ with the synthetic maleimide-modified SpyTag peptide $\left(0.1 \mathrm{mg} \mathrm{ml}^{-1}\right)$ in PBS, then washed in PBS. The electrodes were then incubated for $1 \mathrm{~h}$ with nanobodySpyCatcher fusion protein (anti-GFP, anti-SARS-CoV-1 or anti-MERS-CoV) diluted to $50 \mu \mathrm{M}$ in sensor binding buffer (100 mM HEPES ( $\mathrm{pH} 7.4), 150 \mathrm{mM} \mathrm{NaCl}$, $0.05 \% \mathrm{vol} / \mathrm{vol}$ Tween-20, $0.02 \% \mathrm{wt} / \mathrm{vol} \mathrm{NaN}_{3}$ and $\left.0.1 \% \mathrm{wt} / \mathrm{vol} \mathrm{BSA}\right)$, then rinsed with PBS. Nanobody-functionalized gate electrodes were stored for up to 1 week at $4^{\circ} \mathrm{C}$ in the sensor binding buffer until use.

XPS. XPS analysis was performed using a Kratos AXIS Supra instrument equipped with a monochromatic $\mathrm{Al} \mathrm{K} \alpha \mathrm{X}$-ray source $(1468.6 \mathrm{eV})$. We operated the source at $75 \mathrm{~W}$ under ultra-high vacuum conditions $\left(\sim 10^{-9} \mathrm{mbar}\right)$. The spectra were recorded in a hybrid mode using electrostatic and magnetic lenses and an aperture slot of $300 \times 700 \mu \mathrm{m}$. The survey and high-resolution spectra were acquired at fixed analyzer pass energies of 80 and $20 \mathrm{eV}$, respectively. We mounted the samples in a floating mode to avoid differential charging. The spectra were then acquired under charge neutralization conditions. We calibrated the spectra to a reference of $\mathrm{C} 1 \mathrm{~s}$ at $284.8 \mathrm{eV}$. The Tougaard method was used for background subtraction, which was deconvoluted using Gaussian and Lorentzian methods with XPSPEAK41.

QCM-D. QCM-D measurements were conducted using a QSense analyzer (QE401; Biolin Scientific). The piezoelectrically active gold sensor $\left(0.7854 \mathrm{~cm}^{2}\right)$ was first modified with HDT SAM under the same reaction conditions and then mounted into the QCM-D setup. First, the QCM-D signals, including the change in frequency $(\Delta f)$ and dissipation $(\Delta D)$ were stabilized in PBS. Second, the peptide solution $\left(0.1 \mathrm{mg} \mathrm{ml}^{-1}\right.$ SpyTag peptide in PBS) was injected into the chamber with a flow rate of $100 \mu \mathrm{min}^{-1}$ controlled by a peristaltic pump. After ensuring that the sensor was fully covered with the solution, we stopped the pump for $1 \mathrm{~h}$ and rinsed the sensor surface with PBS injected into the system for $15 \mathrm{~min}$. The same procedure was employed to treat the surface with SpyCatcher-linked nanobody solution ( $50 \mu \mathrm{M}$ in the binding buffer). All QCM-D data presented in this work were recorded at the seventh overtone. To quantify the mass accumulating on the sensor $(\Delta m)$ and the thickness of the layers during the functionalization steps, we used the Sauerbrey equation $(1)^{57}$ :

$$
\Delta m=\frac{-17.7}{n} \Delta f_{n}
$$

where $n$ is the overtone number selected for the calculations and -17.7 is a constant calculated based on the resonant frequency, active area, density and shear modulus of the quartz crystal sensor. The mass of the binding molecules on the sensor surface was then estimated using their molecular weight. The (Sauerbrey) thickness $(\mathrm{nm}), d^{\mathrm{s}}$, was calculated as: 


$$
d^{\mathrm{s}}=\frac{\Delta m}{\operatorname{Ar} 100}
$$

where $\Delta m$ is the change in mass (ng), $A$ is the area $\left(\mathrm{cm}^{2}\right)$ and $r$ is the estimated density of the layer $\left(\mathrm{g} \mathrm{cm}^{-3}\right.$; assumed to be the same as water, which is taken as $\left.1 \mathrm{~g} \mathrm{~cm}^{-3}\right)$.

Electrochemical characterization. The electrochemical characteristics of the gold electrode were investigated before and after the formation of chem-SAM and bio-SAM using EIS and cyclic voltammetry performed in a three-electrode setup using a potentiostat (Autolab PGSTAT128N with Nova software; Metrohm). We used a platinum wire and an $\mathrm{Ag} / \mathrm{AgCl}$ electrode as the counter electrode and reference electrodes, respectively, while the gold electrode was connected as the working electrode. All measurements were carried out in $5 \mathrm{ml}$ of $10 \mathrm{mM}$ PBS (pH 7.4) containing $10 \mathrm{mM}\left[\mathrm{Fe}(\mathrm{CN})_{6}\right]^{3-/ 4-}$. For cyclic the voltammetry measurements, the potential window was between -0.2 and $0.5 \mathrm{~V}$ and the scan rate was $100 \mathrm{mV} \mathrm{s}^{-1}$. Electrochemical impedance spectra were recorded at a d.c. voltage of $0 \mathrm{~V}$ versus the open circuit potential $\left(V_{\text {oc }}\right)$ and an a.c. modulation of $10 \mathrm{mV}$ over a frequency range of $0.1-100,000 \mathrm{~Hz}$. For the analysis of GFP nanobody-functionalized electrodes, the electrodes were incubated with GFP solutions for $10 \mathrm{~min}$ and washed in $10 \mathrm{mM}$ PBS before the measurements. The data were analyzed using Nova software with the appropriate equivalent circuit modeling.

Proteins and peptides. Nanobody-SpyCatcher fusion proteins were designed based on available structures (nanobody: Protein Data Bank (PDB) ID 4PFE ${ }^{58}$; SpyCatcher PDB ID $4 \mathrm{MLI}^{59}$ ) with the nanobody placed at the amino-terminal end of the fusion protein in order to orient the common VHH target-binding interface towards the bulk solution, away from the sensing surface. Protein sequences were reverse-translated and codon-optimized for expression in E. coli with an in-house Python script based on DNA Chisel ${ }^{60}$. Plasmids for protein expression were gene synthesized by Twist Bioscience (United States) or Bio Basic (Canada) in our customized expression vector pJE411c with kanamycin resistance and modified with an RBS insulator (BCD2) cassette ${ }^{61}$ for improved translation initiation. Plasmids were transformed into E. coli BL21 (DE3) and starter cultures were inoculated overnight from a single colony. 11 production cultures in $2 \mathrm{x}$ YT medium with $50 \mathrm{mgl}^{-1} \mathrm{kanamycin}$ and $1 \%$ glucose were inoculated 1:100, grown at $37^{\circ} \mathrm{C}$ and 250 r.p.m. to and optical density measured at $600 \mathrm{~nm}$ of 0.8 , induced with $0.5 \mathrm{mM} \beta$-D-1-thiogalactopyranoside and incubated with shaking for $18 \mathrm{~h}$ at $25^{\circ} \mathrm{C}$. Cells were harvested by centrifugation for $10 \mathrm{~min}$ at $6000 \times g$ and $4^{\circ} \mathrm{C}$, washed once with cold PBS, resuspended in lysis buffer $(25 \mathrm{mM}$ Tris- $\mathrm{HCl}$ ( $\mathrm{pH} 7.4$ ), $500 \mathrm{mM} \mathrm{NaCl}, 10 \mathrm{mM}$ imidazole, $10 \%$ glycerol, SIGMAFAST protease inhibitor, $25 \mathrm{U} \mathrm{ml}^{-1}$ Benzonase HC (Milipore) and $2 \mathrm{mM} \mathrm{DTT}$ ) and homogenized with a cell disruptor (Constant Systems). Earlier purifications of mCherry, GFP nanobody and msfGFP used different lysis methods (chemical lysis with BugBuster (Novagen) for mCherry; sonication for the other two). Lysates were cleared by centrifugation at $87,000 \times g$ for $45 \mathrm{~min}$ and the supernatant was filtered through Miracloth tissue (Milipore) and subjected to affinity chromatography on an ÄKTA FPLC (GE Healthcare) using either StrepTrap HP or HisTrap HP columns (GE Healthcare), depending on the purification tag. The Strep-tag binding buffer was $100 \mathrm{mM}$ Tris- $\mathrm{HCl}$ ( $\mathrm{pH} 8$ ), $150 \mathrm{mM} \mathrm{NaCl}, 1 \mathrm{mM}$ EDTA, 5\% glycerol and $0.5 \mathrm{mM}$ Tris(2 carboxyethyl)phosphine, and elution was performed with $2.5 \mathrm{mM}$ desthiobiotin in binding buffer. The His-tag binding buffer was $25 \mathrm{mM}$ Tris- $\mathrm{HCl}$ (pH 7.4), $500 \mathrm{mM} \mathrm{NaCl}, 10 \mathrm{mM}$ imidazole, $10 \%$ glycerol and $2 \mathrm{mM} \mathrm{DTT}$, and elution was performed with a four-step imidazole gradient up to $0.5 \mathrm{M}$. Fractions were pooled and concentrated using $10 \mathrm{~K}$ Amicon Ultra (Milipore), followed by gel filtration on a Superdex75 16/600 column (GE Healthcare) into $20 \mathrm{mM}$ HEPES ( $\mathrm{pH} 7.5$ ), $300 \mathrm{mM} \mathrm{NaCl}, 10 \%$ glycerol and $50 \mu \mathrm{M}$ EDTA. After spin concentration, aliquots were snap-frozen in liquid nitrogen and stored at $-80^{\circ} \mathrm{C}$. Protein purity, quality and accurate molar mass were monitored by sodium dodecyl sulfate polyacrylamide gel electrophoresis, as well as size-exclusion chromatography multi-angle light scattering on a DAWN HELEOS II and OptiLab T-rEx (Wyatt). Protein concentrations were determined on a NanoDrop spectrophotometer by absorbance at $280 \mathrm{~nm}$ using sequence-specific extinction coefficients (https://web. expasy.org/protparam/).

SARS-CoV-1/2 and MERS target proteins, expressed and purified from HEK293 cells or insect cell culture, were received lyophilized from Sino Biological (China) and dissolved to a standard concentration of $0.25 \mathrm{mg} \mathrm{m}^{-1}$, per the manufacturer's instructions, then aliquoted, snap-frozen in liquid nitrogen and stored at $-80^{\circ} \mathrm{C}$. Amino-terminally maleimide-labeled SpyTag peptides were synthesized by GenScript Biotech (Singapore), received lyophilized, dissolved in PBS and stored at $-20^{\circ} \mathrm{C}$.

Protein dilutions. Target and non-target proteins were thawed on ice and centrifuged at 15,000 r.p.m. at $4^{\circ} \mathrm{C}$ for $30-45 \mathrm{~min}$ to remove any potential aggregates (although no aggregation was observed). Sino Biological proteins were then used as provided for the preparation of dilution series starting at 320 or $640 \mathrm{nM}$. Equivalent dilutions of the Sino Biological storage buffer by itself did not give any sensor response. Weak background sensor signals were recorded from dilutions of DTT. In-house proteins were therefore stored or exchanged into DTT-free buffer before use. The higher-concentrated proteins from in-house production were first diluted to intermediate concentrations that could still be validated and corrected spectrophotometrically. Protein dilutions were prepared in standard sensor binding buffer (100 mM HEPES (pH 7.4), $150 \mathrm{mM} \mathrm{NaCl}, 0.05 \%$ $\mathrm{vol} / \mathrm{vol}$ Tween-20, $0.02 \% \mathrm{wt} / \mathrm{vol} \mathrm{NaN}_{3}$ and $0.1 \% \mathrm{wt} / \mathrm{vol} \mathrm{BSA}$ ), which was modified for saliva spike-in measurements to include complete protease inhibitor cocktail with EDTA (Sigma-Aldrich) at four times the manufacturer-recommended concentration (giving a twofold concentration in the final 1:1 mixture with saliva). BSA was not included in this saliva buffer. For measurements in the regular binding buffer, fourfold dilution series were prepared in 96-well microplates over 23 steps starting from $320 \mathrm{nM}$. For measurements in serum, saliva and UTM, target protein dilution series were prepared in the appropriate buffer (standard or saliva binding buffer) starting from $640 \mathrm{nM}$ so that final concentrations were identical after 1:1 mixture with serum, saliva or UTM.

SPR measurements. SPR measurements were performed on a Biacore T200 instrument using Ni-NTA sensor chips (GE Healthcare) and modified running buffer (20 mM HEPES (pH 7.4), $150 \mathrm{mM} \mathrm{NaCl}, 0.05 \%$ Tween-20, 0.02\% wt/vol $\mathrm{NaN}_{3}$ and $50 \mu \mathrm{M}$ EDTA, prepared at room temperature and filtered) mirroring the sensor binding buffer. All analyte proteins were desalted into the running buffer (HiPrep 26/10 column; GE Healthcare) before the preparation of dilution series in the same running buffer. The GFP nanobody-GFP interaction was measured with the $\mathrm{His}_{10}$-tagged nanobody immobilized (ligand) and msfGFP in solution. For the remaining measurements, since all viral target proteins arrived with a non-cleavable His-tag, target proteins were immobilized instead of the nanobody. All ligand proteins gave stable immobilization responses with minimal signal loss over time. His tags were removed from nanobody-SpyCatcher proteins by overnight cleavage with excess $3 \mathrm{C}$ protease (produced in house), followed by gel filtration on a Superdex 200 Increase 10/300GL column. All ligand proteins were immobilized to equal loading levels of around $100 \mathrm{RU}$ at $10 \mu \mathrm{min}^{-1}$ flow rates. Binding and unbinding experiments were run at a flow rate of $80 \mu \mathrm{l} \mathrm{min}{ }^{-1}$. Two replicates of each dilution series were prepared and measured in the course of the same experiment. Between each measurement cycle, the sensor surface was regenerated with $0.35 \mathrm{M}$ EDTA in running buffer and re-charged with $0.5 \mathrm{mM}$ $\mathrm{NiSO}_{4}$ in water. Biacore results were analyzed with the manufacturer analysis software version 2.1 following standard procedures (double subtraction of the reference channel and buffer injection signal) applying a 1:1 binding model and simultaneous curve fitting. The SARS-CoV-2 RBD results were instead fit to a heterologous ligand binding model.

Fluorescence imaging. Imaging was performed on a DMI8 inverted fluorescence microscope (Leica Microsystems) coupled with a pE-4000 fluorescence illumination system (CoolLED), and the images were processed using ImageJ software. Electrodes were placed between two cover slips in the presence of PBS, to keep the flexible material in plane and in focus. The presence or absence of BSA was studied by adding fluorescently labeled BSA (albumin-fluorescein isothiocyanate conjugated bovine protein) to the nanobody-SpyCatcher solution during functionalization of the sensing surface. The capture of GFP was examined by imaging already functionalized gold electrodes before and after incubation with GFP or control solutions.

OECT characterization and sensor operation. We used a Keithley Source Meter operating by LabVIEW code to apply drain $\left(V_{\mathrm{D}}\right)$ and gate voltages $\left(V_{\mathrm{G}}\right)$ and to obtain gate and channel currents $\left(I_{\mathrm{G}}\right.$ and $\left.I_{\mathrm{D}}\right)$ under ambient conditions. A polydimethylsiloxane well was glued on top of the channels and filled with $75 \mu \mathrm{l}$ of $10 \mathrm{mM}$ PBS. The steady-state transistor characteristics were obtained by measuring $I_{\mathrm{D}}$ versus $V_{\mathrm{D}}$ at various $V_{\mathrm{G}}$, for PEDOT:PSS applied between -0.6 and $0.6 \mathrm{~V}$ with $0.1-\mathrm{V}$ steps $\left(2.5 \mathrm{~V} \mathrm{~s}^{-1}\right)$, and for $\mathrm{p}(\mathrm{g} 0 \mathrm{~T} 2$-g6 2 ) from 0.2 to $-0.6 \mathrm{~V}$ with $0.05-\mathrm{V}$ steps $\left(1.25 \mathrm{~V} \mathrm{~s}^{-1}\right) . V_{D}$ was swept from 0 to $-0.6 \mathrm{~V}$. We first chose a channel and obtained its transfer curve $\left(I_{\mathrm{D}}\right.$ versus $\left.V_{\mathrm{G}}\right)$ in PBS using the nanobody-functionalized gate electrode incubated for $10 \mathrm{~min}$ in the buffer solution (buffer, saliva, UTM or serum, in the absence of target proteins). The currents obtained were used as the baseline signal $\left(I_{0}\right)$. The same gate electrode was then incubated with $5 \mu \mathrm{l}$ of the solution (buffer, saliva, UTM or serum) containing the protein target for $10 \mathrm{~min}$ (pipetting for $30 \mathrm{~s}$ every $3 \mathrm{~min}$ ). The electrode was washed thoroughly with PBS to remove any unbound proteins. To obtain calibration curves, we prepared various concentrations of the target protein and collected device data starting from the most diluted one. The normalized response of the sensor was calculated according to the following equation:

$$
\text { Normalized response }=\frac{\left|I_{\mathrm{D}}-I_{0}\right|}{I_{0}}
$$

where $I_{\mathrm{D}}$ is the current response of the sensor to an analyte solution that the gate was exposed to.

According to the International Union of Pure and Applied Chemistry, the LOD was calculated as the concentration leading to a response that equalled the average of the noise level plus three times the noise standard deviation ${ }^{13,62}$. The level of 
noise $\left(\frac{\Delta I}{I_{0}} \pm 3 \delta\right)$ was taken from the relative current variation in negative control sensors.

$$
\operatorname{LOD}=\frac{\left(\frac{\Delta I}{I_{0}} \pm 3 \delta\right)-a}{b}
$$

where $\frac{\Delta I}{I_{0}}$ is the average response of the blank sample, $\delta$ is the relative standard deviation and $a$ and $b$ are the intercept and slope of the calibration curve, respectively.

Clinical sample preparation and testing. The clinical samples (saliva samples and nasopharyngeal swabs) used in this study (Fig. 5) were collected from human subjects as part of registered protocols approved by the Institutional Review Board of the King Faisal Specialist Hospital and Research Center and King Abdullah University of Science and Technology (KAUST) Institutional Biosafety and Bioethics Committee (IBEC). All volunteers provided signed consent to participate in the study. Nasopharyngeal swabs collected from outpatients with COVID19 and healthy subjects were stored in UTM at $4{ }^{\circ} \mathrm{C}$ and tested within $1-2 \mathrm{~d}$ of collection. Saliva was collected in $50-\mathrm{ml}$ tubes and always measured the same day. The saliva used for original sensor characterization with recombinant proteins (Fig. 4) was self-collected in the morning before food or tooth brushing, filtered and stored in aliquots at $-20^{\circ} \mathrm{C}$. All volunteers provided signed consent to participate in the study and provide saliva. The buffer composition for measurements in clinical samples was modified in the course of the study: nasal swabs were measured after 1:1 dilution in the original buffer used for the saliva spike-in experiments (100 mM HEPES ( $\mathrm{pH} 7.4$ ), $150 \mathrm{mM} \mathrm{NaCl}, 0.05 \%$ vol/vol Tween-20, $0.02 \% \mathrm{wt} / \mathrm{vol} \mathrm{NaN}_{3}$ and $4 \mathrm{x}$ cOmplete). Raw saliva samples were measured after 1:3 dilution in a virus-inactivating lysis buffer (50 mM Tris (pH 7.4), $250 \mathrm{mM} \mathrm{NaCl}, 1 \%$ Nonidet P-40, $0.02 \% \mathrm{NaN}_{3}, 0.5 \%$ BSA and $4 \mathrm{x}$ cOmplete). Human male AB plasma (serum) was purchased from Sigma-Aldrich and used as received. All protocols and procedures involving human saliva, nasopharyngeal swabs and serum were approved by the KAUST IBEC (under approval numbers 18IBEC11 and 20IBEC25) and National Committee of BioEthics, Saudi Arabia (registration number HAP02-J-042). The study conducted at the hospital was approved by the Office of Research Affairs of the King Faisal Specialist Hospital and Research Centre (RAC \#220 0014).

Reporting Summary. Further information on research design is available in the Nature Research Reporting Summary linked to this article.

\section{Data availability}

The data supporting the results of this study are available within the paper and its Supplementary Information. All of the data generated during this study and used to produce the figures are available from Figshare at https://doi.org/10.6084/ m9.figshare.14420588.

Received: 10 November 2020; Accepted: 16 April 2021; Published online: 24 May 2021

\section{References}

1. Kissler, S. M., Tedijanto, C., Goldstein, E., Grad, Y. H. \& Lipsitch, M. Projecting the transmission dynamics of SARS-CoV-2 through the postpandemic period. Science 368, 860-868 (2020)

2. Lewis, D. Mounting evidence suggests coronavirus is airborne-but health advice has not caught up. Nature 583, 510-513 (2020).

3. Oran, D. P. \& Topol, E. J. Prevalence of asymptomatic SARS-CoV-2 infection. Ann. Intern. Med. https://doi.org/10.7326/M20-3012 (2020).

4. Long, Q.-X. et al. Clinical and immunological assessment of asymptomatic SARS-CoV-2 infections. Nat. Med. 26, 1200-1204 (2020).

5. WHO. WHO Director-General's Opening Remarks at the Media Briefing on COVID-19-16 March 2020 (World Health Organization, 2020); https://www. who.int/dg/speeches/detail/who-director-general-s-opening-remarks-at-themedia-briefing-on-covid-19---16-march-2020

6. Corman, V. M. et al. Detection of 2019 novel coronavirus (2019-nCoV) by real-time RT-PCR. Eurosurveillance 25, 2000045 (2020).

7. Ferretti, L. et al. Quantifying SARS-CoV-2 transmission suggests epidemic control with digital contact tracing. Science 368, eabb6936 (2020).

8. Krueger, L. J. et al. Evaluation of the accuracy, ease of use and limit of detection of novel, rapid, antigen-detecting point-of-care diagnostics for SARS-CoV-2. Preprint at medRxiv https://doi. org/10.1101/2020.10.01.20203836 (2020).

9. Niemz, A., Ferguson, T. M. \& Boyle, D. S. Point-of-care nucleic acid testing for infectious diseases. Trends Biotechnol. 29, 240-250 (2011).

10. Wang, W. U., Chen, C., Lin, K., Fang, Y. \& Lieber, C. M. Label-free detection of small-molecule-protein interactions by using nanowire nanosensors. Proc. Natl Acad. Sci. USA 102, 3208-3212 (2005).

11. Ren, R. et al. Nanopore extended field-effect transistor for selective single-molecule biosensing. Nat. Commun. 8, 586 (2017).
12. Nakatsuka, N. et al. Aptamer-field-effect transistors overcome Debye length limitations for small-molecule sensing. Science 362, 319-324 (2018).

13. Macchia, E. et al. Single-molecule detection with a millimetre-sized transistor. Nat. Commun. 9, 3223 (2018).

4. Macchia, E. et al. About the amplification factors in organic bioelectronic sensors. Mater. Horiz. 7, 999-1013 (2020).

15. Khodagholy, D. et al. High transconductance organic electrochemical transistors. Nat. Commun. 4, 2133 (2013).

16. Inal, S., Rivnay, J., Suiu, A.-O., Malliaras, G. G. \& McCulloch, I. Conjugated polymers in bioelectronics. Acc. Chem. Res. 51, 1368-1376 (2018).

17. Rivnay, J. et al. Organic electrochemical transistors. Nat. Rev. Mater. 3, 17086 (2018).

18. Ohayon, D. \& Inal, S. Organic bioelectronics: from functional materials to next-generation devices and power sources. Adv. Mater. 32, 2001439 (2020).

19. Gentili, D. et al. Integration of organic electrochemical transistors and immuno-affinity membranes for label-free detection of interleukin-6 in the physiological concentration range through antibody-antigen recognition. J. Mater. Chem. B 6, 5400-5406 (2018).

20. Macchia, E. et al. Ultra-sensitive protein detection with organic electrochemical transistors printed on plastic substrates. Flex. Print. Electron. 3, 034002 (2018).

21. Trilling, A. K., Beekwilder, J. \& Zuilhof, H. Antibody orientation on biosensor surfaces: a minireview. Analyst 138, 1619-1627 (2013).

22. Muyldermans, S. Nanobodies: natural single-domain antibodies. Annu. Rev. Biochem. 82, 775-797 (2013).

23. Hamers-Casterman, C. et al. Naturally occurring antibodies devoid of light chains. Nature 363, 446-448 (1993).

24. Steeland, S., Vandenbroucke, R. E. \& Libert, C. Nanobodies as therapeutics: big opportunities for small antibodies. Drug Discov. Today 21, 1076-1113 (2016)

25. De Meyer, T., Muyldermans, S. \& Depicker, A. Nanobody-based products as research and diagnostic tools. Trends Biotechnol. 32, 263-270 (2014).

26. Filipiak, M. S. et al. Highly sensitive, selective and label-free protein detection in physiological solutions using carbon nanotube transistors with nanobody receptors. Sens. Actuators B Chem. 255, 1507-1516 (2018).

27. Rothbauer, U. et al. A versatile nanotrap for biochemical and functional studies with fluorescent fusion proteins. Mol. Cell. Proteom. 7, 282-289 (2008).

28. Zakeri, B. et al. Peptide tag forming a rapid covalent bond to a protein, through engineering a bacterial adhesin. Proc. Natl Acad. Sci. USA 109, E690-E697 (2012).

29. Keeble, A. H. \& Howarth, M. Insider information on successful covalent protein coupling with help from SpyBank. Methods Enzymol. 617, 443-461 (2019).

30. Oloketuyi, S. et al. Electrochemical immunosensor functionalized with nanobodies for the detection of the toxic microalgae Alexandrium minutum using glassy carbon electrode modified with gold nanoparticles. Biosens. Bioelectron. 154, 112052 (2020).

31. Esplandiu, M. J. \& Noeske, P.-L. M. XPS investigations on the interactions of 1,6-hexanedithiol/Au(111) layers with metallic and ionic silver species. Appl. Surf. Sci. 199, 166-182 (2002).

32. Love, J. C., Estroff, L. A., Kriebel, J. K., Nuzzo, R. G. \& Whitesides, G. M. Self-assembled monolayers of thiolates on metals as a form of nanotechnology. Chem. Rev. 105, 1103-1170 (2005).

33. Weser, U. in Cation Ordering and Electron Transfer (eds Gleitzer, C. et al.) 145-160 (Springer, 1985).

34. Zhang, S., Garcia-D’Angeli, A., Brennan, J. P. \& Huo, Q. Predicting detection limits of enzyme-linked immunosorbent assay (ELISA) and bioanalytical techniques in general. Analyst 139, 439-445 (2013).

35. Moser, M., Ponder, J. F., Wadsworth, A., Giovannitti, A. \& McCulloch, I. Materials in organic electrochemical transistors for bioelectronic applications: past, present, and future. Adv. Funct. Mater. 29, 1807033 (2019).

36. Moser, M. et al. Side chain redistribution as a strategy to boost organic electrochemical transistor performance and stability. Adv. Mater. 32, 2002748 (2020)

37. Venkatraman, V. et al. Subthreshold operation of organic electrochemical transistors for biosignal amplification. Adv. Sci. 5, 1800453 (2018).

38. Macchia, E. et al. Label-free and selective single-molecule bioelectronic sensing with a millimeter-wide self-assembled monolayer of anti-immunoglobulins. Chem. Mater. 31, 6476-6483 (2019).

39. Kubala, M. H., Kovtun, O., Alexandrov, K. \& Collins, B. M. Structural and thermodynamic analysis of the GFP:GFP-nanobody complex. Protein Sci. 19, 2389-2401 (2010).

40. Schuck, P. \& Zhao, H. The role of mass transport limitation and surface heterogeneity in the biophysical characterization of macromolecular binding processes by SPR biosensing. Methods Mol. Biol. 627, 15-54 (2010).

41. Sheehan, P. E. \& Whitman, L. J. Detection limits for nanoscale biosensors. Nano Lett. 5, 803-807 (2005).

42. Macchia, E., Manoli, K., Di Franco, C., Scamarcio, G. \& Torsi, L. New trends in single-molecule bioanalytical detection. Anal. Bioanal. Chem. 412, 5005-5014 (2020) 
43. Wrapp, D. et al. Structural basis for potent neutralization of betacoronaviruses by single-domain Camelid antibodies. Cell 181, 1004-1015.e15 (2020).

44. Raj, S. V. et al. Chimeric camel/human heavy-chain antibodies protect against MERS-CoV infection. Sci. Adv. 4, eaas9667 (2018).

45. Walls, A. C. et al. Structure, function, and antigenicity of the SARS-CoV-2 spike glycoprotein. Cell 181, 281-292.e6 (2020)

46. Hanke, L. et al. An alpaca nanobody neutralizes SARS-CoV-2 by blocking receptor interaction. Nat. Commun. 11, 4420 (2020).

47. Pasomsub, E. et al. Saliva sample as a non-invasive specimen for the diagnosis of coronavirus disease 2019: a cross-sectional study. Clin. Microbiol. Infect. 27, 285.E1-285.E4 (2021).

48. Wyllie, A. L. et al. Saliva or nasopharyngeal swab specimens for detection of SARS-CoV-2. N. Engl. J. Med. 383, 1283-1286 (2020).

49. Butler-Laporte, G. et al. Comparison of saliva and nasopharyngeal swab nucleic acid amplification testing for detection of SARS-CoV-2: a systematic review and meta-analysis. JAMA Intern. Med. 181, 353-360 (2021)

50. Azhar, E. I., Hui, D. S. C., Memish, Z. A., Drosten, C. \& Zumla, A. The Middle East respiratory syndrome (MERS). Infect. Dis. Clin. 33, 891-905 (2019)

51. Todd, J. et al. Ultrasensitive flow-based immunoassays using single-molecule counting. Clin. Chem. 53, 1990-1995 (2007).

52. Spitzberg, J. D., Zrehen, A., van Kooten, X. F \& Meller, A. Plasmonic-nanopore biosensors for superior single-molecule detection. Adv. Mater. 31, 1900422 (2019).

53. Alexandersen, S., Chamings, A. \& Bhatta, T. R. SARS-CoV-2 genomic and subgenomic RNAs in diagnostic samples are not an indicator of active replication. Nat. Commun. 11, 6059 (2020).

54. Ohayon, D. et al. Biofuel powered glucose detection in bodily fluids with an n-type conjugated polymer. Nat. Mater. 19, 456-463 (2020).

55. Wustoni, S., Savva, A., Sun, R., Bihar, E. \& Inal, S. Enzyme-free detection of glucose with a hybrid conductive gel electrode. Adv. Mater. Interfaces 6, 1800928 (2019).

56. $\mathrm{Qu}, \mathrm{D}$. et al. 1,6-Hexanedithiol self-assembled monolayers on $\mathrm{Au}(111)$ investigated by electrochemical, spectroscopic, and molecular mechanics methods. J. Phys. Chem. C. 114, 497-505 (2010).

57. Höök, F., Rodahl, M., Brzezinski, P. \& Kasemo, B. Energy dissipation kinetics for protein and antibody-antigen adsorption under shear oscillation on a quartz crystal microbalance. Langmuir 14, 729-734 (1998).

58. Eshaghi, M. et al. Rational structure-based design of bright GFP-based complexes with tunable dimerization. Angew. Chem. Int. Ed. Engl. 54, 13952-13956 (2015)

59. Li, L., Fierer, J. O., Rapoport, T. A. \& Howarth, M. Structural analysis and optimization of the covalent association between SpyCatcher and a peptide Tag. J. Mol. Biol. 426, 309-317 (2014).

60. Zulkower, V. \& Rosser, S. DNA Chisel, a versatile sequence optimizer. Bioinformatics 36, 4508-4509 (2020).

61. Mutalik, V. K. et al. Precise and reliable gene expression via standard transcription and translation initiation elements. Nat. Methods 10, 354-360 (2013).

62. Long, G. L. \& Winefordner, J. D. Limit of detection. A closer look at the IUPAC definition. Anal. Chem. 55, 712A-724A (1983).

\section{Acknowledgements}

Figure 1 was produced by X. Pita, a scientific illustrator at KAUST. We thank all of the members of the KAUST Rapid Research Response Team (R3T) for COVID-19, especially S. Hamdan, for contributions in this study. We thank S. Mfarrej and A. K. Subudhi for providing access to and assisting with the experiments in the Biosafety Level 2+ experimental room at KAUST. We thank the KAUST Health team (operated by Dr. Soliman Fakeeh Hospital, Jeddah), including D. Buttigieg and M. Habib, for providing clinical samples. We thank staff at the King Faisal Specialist Hospital and Research Center (Riyadh), particularly A. Alzahrani, M. Alsanea and F. Alhadeq, for help with organizing and hosting some of the clinical studies. We thank the KAUST nanofabrication core laboratory team, D. Rosas Villalva and U. Buttner for help with device fabrication and integration. This work was initiated thanks to the KAUST Impact Acceleration Fund (IAF) program. The research reported in this publication was supported by funding from the Office of Sponsored Research (OSR) at KAUST under award numbers REI/1/4204-01, REI/1/4229-01, OSR-2018-CRG7-3709, OSR-2018-CARF/CCF-3079, OSR-2015-CRG4-2572 and OSR-4106 CPF2019. We acknowledge EC FP7 Project SC2 (610115), EC H2020 (643791) and EPSRC Projects EP/ G037515/1, EP/M005143/1 and EP/L016702/1.

\section{Author contributions}

S.I., S.T.A. and R.G. conceived of the research, designed the experiments, supervised the work and wrote the manuscript. K.G., S.W. and A.K. fabricated the devices and performed the OECT experiments. K.G. and S.W. functionalized the gate electrodes. A.K. performed the EIS measurements and conducted the modelling. S.W. performed the cyclic voltammetry and XPS measurements. K.G. conducted the QCM-D experiments. R.G. and E.D.-G. designed and produced the recombinant proteins and performed the Biacore experiments. A.H. took the fluorescence microscope images and developed the LabVIEW codes to operate the OECTs. A.N.A. collected the clinical samples. M.S. and A.P. provided the RT-qPCR results. F.S.A. supervised the nasal swab collection and conducted the RT-PCR with A.A.A. and R.G. M.M. and I.M. provided the p-type material. All authors were involved in the discussion and participated in manuscript input.

\section{Competing interests}

S.I., S.T.A., R.G., K.G., S.W. and A.K. have filed a patent application (US Patent application no. 63/047,547; "Nanobody-functionalized electrochemical transistors and methods of making and using thereof") related to this work.

\section{Additional information}

Supplementary information The online version contains supplementary material available at https://doi.org/10.1038/s41551-021-00734-9.

Correspondence and requests for materials should be addressed to S.T.A., R.G. or S.I.

Peer review information Nature Biomedical Engineering thanks Howard Katz and the other, anonymous, reviewer(s) for their contribution to the peer review of this work. Peer reviewer reports are available.

Reprints and permissions information is available at www.nature.com/reprints. Publisher's note Springer Nature remains neutral with regard to jurisdictional claims in published maps and institutional affiliations.

(C) The Author(s), under exclusive licence to Springer Nature Limited 2021 


\section{Reporting Summary}

Nature Research wishes to improve the reproducibility of the work that we publish. This form provides structure for consistency and transparency in reporting. For further information on Nature Research policies, see our Editorial Policies and the Editorial Policy Checklist.

\section{Statistics}

For all statistical analyses, confirm that the following items are present in the figure legend, table legend, main text, or Methods section.

n/a Confirmed

$\bigotimes$ The exact sample size $(n)$ for each experimental group/condition, given as a discrete number and unit of measurement

$\searrow$ A statement on whether measurements were taken from distinct samples or whether the same sample was measured repeatedly

$\square$ The statistical test(s) used AND whether they are one- or two-sided

$\triangle \square$ Only common tests should be described solely by name; describe more complex techniques in the Methods section.

Х $\square$ A description of all covariates tested

Х $\square$ A description of any assumptions or corrections, such as tests of normality and adjustment for multiple comparisons

$\square$ A full description of the statistical parameters including central tendency (e.g. means) or other basic estimates (e.g. regression coefficient)

$\triangle \square$ AND variation (e.g. standard deviation) or associated estimates of uncertainty (e.g. confidence intervals)

$\triangle \square$ For null hypothesis testing, the test statistic (e.g. $F, t, r$ ) with confidence intervals, effect sizes, degrees of freedom and $P$ value noted

$\triangle$ Give $P$ values as exact values whenever suitable.

Х $\square$ For Bayesian analysis, information on the choice of priors and Markov chain Monte Carlo settings

Х $\square$ For hierarchical and complex designs, identification of the appropriate level for tests and full reporting of outcomes

\ $\square$ Estimates of effect sizes (e.g. Cohen's $d$, Pearson's $r$ ), indicating how they were calculated

Our web collection on statistics for biologists contains articles on many of the points above.

\section{Software and code}

Policy information about availability of computer code

Data collection LabView 2017; Nova 2.1.3 (MetroOhm); QSoft401 v. 2.8.4 (Biolin Scientific).

Data analysis Nova 2.1.3 (MetroOhm) for impedance fitting; XPSPEAK 4.1 for XPS data analysis; ImageJ 1.53c for fluorescence image analysis.

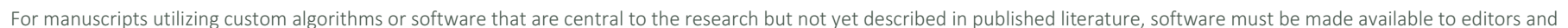

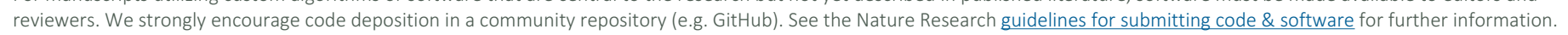

\section{Data}

Policy information about availability of data

All manuscripts must include a data availability statement. This statement should provide the following information, where applicable:

- Accession codes, unique identifiers, or web links for publicly available datasets

- A list of figures that have associated raw data

- A description of any restrictions on data availability

The data supporting the results in this study are available within the paper and its Supplementary Information. All the data generated during the study to make the figures are available from figshare at http://doi.org/10.6084/m9.figshare.14420588. 


\section{Field-specific reporting}

Please select the one below that is the best fit for your research. If you are not sure, read the appropriate sections before making your selection.

\Life sciences

Behavioural \& social sciences

Ecological, evolutionary \& environmental sciences

For a reference copy of the document with all sections, see nature.com/documents/nr-reporting-summary-flat.pdf

\section{Life sciences study design}

All studies must disclose on these points even when the disclosure is negative.

Sample size No sample-size calculations were performed. We did not quantify sensor specificity or sensitivity. Appropriate large-scale clinical studies were out of the scope of the current stage of development (see also 'Randomization' below).

Data exclusions We excluded the sensor-validation results of one nasal swab sample from one participant because the negative control gate (GFP nanobody) gave an unusually high signal. Although the actual sensor (with SARS gate) tested the sample as COVID-19-positive (as confirmed by PCR), we did not include these data because of the control measurements. Control sensors otherwise confirmed the quality of all other patient samples presented in this work and thus the reliability of the actual sensors.

Replication Data of all replicates are included in the manuscript.

Randomization Randomization was not performed. Fresh saliva samples from early-stage infections were only available to us once (collected by KAUST Health from patient's homes in the wake of a campus-wide PCR testing campaign), and we opted to focus our restricted measurement capacity on these samples. Randomized and blinded tests will be performed as device development moves closer to practical application and product design.

Blinding No blinding was performed (see also 'Randomization' above).

\section{Reporting for specific materials, systems and methods}

We require information from authors about some types of materials, experimental systems and methods used in many studies. Here, indicate whether each material, system or method listed is relevant to your study. If you are not sure if a list item applies to your research, read the appropriate section before selecting a response.

Materials \& experimental systems

$\mathrm{n} / \mathrm{a}$ Involved in the study

Methods

$\square$ \ Antibodies

\ $\square$ Eukaryotic cell lines

$\bigotimes \square$ Palaeontology and archaeology

$\mathrm{n} / \mathrm{a}$ Involved in the study

X $\square$ chIP-seq

$\triangle \square$ Animals and other organisms

X $\square$ Flow cytometry

Х $\square$ MRI-based neuroimaging

$\square$ \uman research participants

\ $\square$ Clinical data

\ $\square$ Dual use research of concern

\section{Antibodies}

Antibodies used

All nanobody sequences were obtained from relevant publications, and the proteins were produced in our lab.

Validation

Validation data are provided in the Supplementary Information (SPR characterization).

\section{Human research participants}

\section{Policy information about studies involving human research participants}

Population characteristics

Recruitment of study participants is described below and in the manuscript. Only minimal details (positive or negative SARSCoV-2 test status, and date of symptom onset or of asymptomatic status) were shared with researchers. Given the small number of available samples (in particular, positive samples), no attempt was made to sample any particular population.

Recruitment

The human research participants were walk-in patients of King Faisal Specialist Hospital \& Research Center, or they were patients (healthy or just diagnosed with SARS-CoV-2 in a campus-wide PCR testing campaign) who volunteered to give samples to KAUST Health. All volunteers provided consent to participate in the study.

Ethics oversight

All protocols and procedures involving human saliva, nasopharyngeal swabs and serum were approved by King Abdullah 
